\title{
Pacific
}

Journal of

Mathematics

\section{A REGULARITY THEOREM FOR GRAPHIC SPACELIKE MEAN CURVATURE FLOWS}

\author{
BENJAMIN STUART THORPE
}




\title{
A REGULARITY THEOREM FOR GRAPHIC SPACELIKE MEAN CURVATURE FLOWS
}

\author{
BENJAMIN STUART THORPE
}

\begin{abstract}
For mean curvature flows in Euclidean spaces, Brian White proved a regularity theorem which gives $C^{2, \alpha}$ estimates in regions of spacetime where the Gaussian density is close enough to 1 . This is proved by applying Huisken's monotonicity formula. Here we will consider mean curvature flows in semiEuclidean spaces, where each spatial slice is an $\boldsymbol{m}$-dimensional graph in $\mathbb{R}_{n}^{m+n}$ satisfying a gradient bound stronger than the spacelike condition. By defining a suitable quantity to replace the Gaussian density ratio, we prove monotonicity theorems similar to Huisken's and use them to prove a regularity theorem similar to White's.
\end{abstract}

\section{Introduction}

A mean curvature flow can roughly be described as a family of submanifolds $\mathcal{M}=\{M(t)\}_{t \in I}$ evolving with velocity equal to the mean curvature vector on each $M(t)$. Let $M$ be such a flow, where each spatial slice $M(t)$ is assumed to be an $m$-dimensional submanifold of a Euclidean space. For spacetime points $(y, s)$, the Gaussian density ratio is given by

$$
\int_{M(t)} \frac{1}{(4 \pi(s-t))^{m / 2}} \exp \left(-\frac{|x-y|^{2}}{4(s-t)}\right) d x
$$

for times $t<s$. Huisken [1990] proved an important monotonicity formula, which roughly says that this will be nonincreasing with respect to $t$ on mean curvature flows. A local version of this formula was proved by Ecker [2004, Proposition 4.17]. One application of these monotonicity formulas is the proof of Brian White's [2005] local regularity theorem for mean curvature flows in Euclidean spaces. This theorem says that such a flow will be smooth in regions of spacetime where the Gaussian density ratios are close enough to 1 .

Our goal is to prove a similar regularity theorem, but now for spacelike mean curvature flows in semi-Euclidean spaces. We will assume that these flows are

MSC2010: 35B65, 35K93.

Keywords: mean curvature flow, semi-Riemannian geometry. 
graphs and that they satisfy some uniform gradient bound stronger than the spacelike condition. Roughly, we will prove that if such a flow is smooth on an interval $(0, T)$ then it can be extended smoothly to time $T$ (see Theorem 16). This should be compared to [White 2005, Theorem 3.5]. We prove this by defining a quantity that has similar properties to the Gaussian density ratio. This quantity is chosen in such a way that the evolution equations for spacelike mean curvature flows will allow us to prove monotonicity formulas similar to Huisken's and Ecker's. The proof of the regularity theorem itself is then similar to the proofs in [White 2005] and [Ecker 2004], with some adjustments.

The main differences between this case and the Euclidean case are caused by the semi-Euclidean metric. Obviously the mean curvature flow system is only parabolic when the spacelike condition is satisfied. Therefore any gradient estimates are only useful if they are stronger than the spacelike condition. This is why we will always assume such a bound on the gradient. ${ }^{1}$ This assumption is also useful when defining our replacement for the Gaussian density ratio. For example, we need the gradient bound to guarantee that this quantity is finite on a smooth flow (since we need the eigenvalues of the induced metric to stay uniformly away from zero). We will frequently need this assumption, used with inequality (4), to get the uniform bounds needed to use the dominated convergence theorem (such arguments here are more difficult than in the Euclidean case, and therefore will be explained in more detail).

Other difficulties due to the semi-Euclidean metric appear in the proofs of the monotonicity and regularity theorems. For example, Ecker's local formula involves a nice localisation function which is not useful in the semi-Euclidean case, thus making our proof of local monotonicity slightly more awkward (see Theorem 10 and compare to [Ecker 2004, Proposition 4.17]). We also get different signs in the evolution equations for various quantities, so that the inequalities seen in the Euclidean case are often reversed here (see Equation (8), for example). The results of this are seen in the monotonicity theorems, where we see that certain quantities are nondecreasing, but where the corresponding quantities in the Euclidean case would be nonincreasing (also see Theorem 13, where the inequality in the assumption is the reverse of what we get in the Euclidean case).

The results proved in this paper formed part of the author's Ph.D. thesis at Durham University, under the supervision of Wilhelm Klingenberg.

\section{Preliminaries}

Notation. We will attempt to keep our notation as close to the notation in [White 2005] as possible, so that the similarities are clear. When $N \geq 2, \mathbb{R}^{N}$ will be

\footnotetext{
${ }^{1}$ For a case where such an estimate holds, see the appendix of [Thorpe 2011].
} 
Euclidean space with elements denoted by $x$ and with the usual norm $|x| . B_{R}^{N}(x)$ will be the ball of radius $R$ and centre $x$. We will denote by $\mathbb{R}^{N, 1}$ the spacetime $\mathbb{R}^{N} \times \mathbb{R}$ with elements $X=(x, t)$ and parabolic norm $\|X\|=\max \left\{|x|,|t|^{1 / 2}\right\}$. We write $B_{R}^{N, 1}(X)=B_{R}^{N}(x) \times\left(t-R^{2}, t+R^{2}\right)$ and $U_{R}^{N, 1}(X)=B_{R}^{N}(x) \times\left(t-R^{2}, t\right]$. The function $\tau: \mathbb{R}^{N, 1} \rightarrow \mathbb{R}$ will be the projection $\tau(x, t)=t$ onto the time axis. For any $\lambda>0$, we define the parabolic dilation $D_{\lambda}: \mathbb{R}^{N, 1} \rightarrow \mathbb{R}^{N, 1}$ by $D_{\lambda}(x, t)=\left(\lambda x, \lambda^{2} t\right)$. Note that $\left\|D_{\lambda} X\right\|=\lambda\|X\|$. For subsets $U$ of $\mathbb{R}^{N, 1}$ and functions $f$ from $U$ into some Euclidean space, we define

$$
d(X, U)=\inf \{\|X-Y\| \mid Y \notin U\} \quad \text { and } \quad\|f\|_{p, \alpha}=\sum_{k+2 h \leq p}\left\|D^{k}\left(\partial_{t}\right)^{h} f\right\|_{0, \alpha},
$$

(for $0<\alpha<1$ and nonnegative integers $p$ ) where $[f]_{\alpha}=\sup _{X \neq Y \in U} \mid f(X)-$ $f(Y) \mid /\|X-Y\|^{\alpha}$ and $\|f\|_{0, \alpha}=\sup _{U}|f|+[f]_{\alpha}$, and where we have used the notation $\partial_{t} f=\partial f / \partial t, \partial_{A} f=\partial f / \partial x^{A}, D=\left(\partial_{1}, \ldots, \partial_{N}\right)$. In the obvious way, we also define the parabolic $C^{p}$ norm by $\|f\|_{p}=\sum_{k+2 h \leq p} \sup _{U}\left|D^{k}\left(\partial_{t}\right)^{h} f\right|$. If we say that a sequence of functions converges in $C^{p}$ or $C^{p, \alpha}$ on some set, we just mean that it converges on that set with respect to the corresponding norm.

Semi-Euclidean spaces. For integers $m \geq 2$ and $n \geq 1$, it will be convenient here for us to consider the space $\mathbb{R}^{m+n}$ with elements denoted by $x=(\hat{x}, \tilde{x})$, where $\hat{x} \in \mathbb{R}^{m}$ and $\tilde{x} \in \mathbb{R}^{n}$. With this notation, we can define the semi-Euclidean spaces $\mathbb{R}_{n}^{m+n}=\left(\mathbb{R}^{m+n},\langle\cdot, \cdot\rangle\right)$ with metric tensor $\langle x, y\rangle=\hat{x} \cdot \hat{y}-\tilde{x} \cdot \tilde{y}$. If we use the summation convention with indices $i, j=1, \ldots, m$ and $v, \gamma=m+1, \ldots, m+n$, then $\langle x, y\rangle=x^{i} y^{i}-x^{\gamma} y^{\gamma}$ and we denote by $\bar{g}$ the corresponding diagonal matrix with $\bar{g}_{i j}=\delta_{i j}, \bar{g}_{\nu \gamma}=-\delta_{\nu \gamma}$.

Let $M$ be a submanifold of $\mathbb{R}_{n}^{m+n}$; then we can take the induced metric $g$ on $M$ in the usual way, and we say that $M$ is spacelike if $g$ is positive definite. The corresponding Levi-Civita connections (denoted $\bar{\nabla}$ and $\nabla$ ) are defined in the usual way, and the second fundamental form on $M$ is given by $B(V, W)=\bar{\nabla}_{V} W-\nabla_{V} W=$ $\left(\bar{\nabla}_{V} W\right)^{\perp}$ for tangent vector fields $V, W$ on $M$ (where $\perp$ denotes projection to normal spaces of $M$ in $\mathbb{R}_{n}^{m+n}$ ). Taking the trace of this (with respect to the induced metric $g$ ) gives the mean curvature vector $H=\operatorname{trace}_{g} B$ of this submanifold. We can also define the gradient $\left(\operatorname{grad}_{M}\right)$, divergence $\left(\operatorname{div}_{M}\right)$ and induced Laplace operator $\left(\Delta_{M}\right)$ on this submanifold, all taken with respect to the induced metric; see [O’Neill 1983] for details.

If $\Omega$ is a domain in $\mathbb{R}^{m}$ and $F: \Omega \rightarrow \mathbb{R}_{n}^{m+n}$ is an embedding such that $M=F(\Omega)$ is a spacelike submanifold of $\mathbb{R}_{n}^{m+n}$, then it is not difficult to check that the mean curvature is given by

$$
H=\left(g^{i j} \partial_{i j} F\right)^{\perp}=\frac{1}{\sqrt{\operatorname{det} g}} \partial_{i}\left(\sqrt{\operatorname{det} g} g^{i j} \partial_{j} F\right)=\Delta_{M} F,
$$


where $g_{i j}=\left\langle\partial_{i} F, \partial_{j} F\right\rangle$ gives the induced metric. This is proved as in the Euclidean case; see [Ecker 2004, Appendix A].

\section{Graphic mean curvature flows}

We will consider graphic flows of the form

$$
M=\{(\hat{x}, u(\hat{x}, t), t) \mid \hat{x} \in \Omega, t \in I\} \subset \mathbb{R}_{n}^{m+n} \times \mathbb{R},
$$

where $\Omega$ is some domain in $\mathbb{R}^{m}, I$ is some time interval in $\mathbb{R}$ (not necessarily open) and $u: \Omega \times I \rightarrow \mathbb{R}^{n}$. When we say that such a flow $\mathcal{M}$ is smooth (or locally $C^{2, \alpha}$, etc.), we mean that the function $u$ has that property. We will also discuss sequences $\mu_{J}$ of such flows (where $J=1,2, \ldots$ ). When we talk about convergence of $\mu_{J}$ in some space of functions, we actually mean convergence of the corresponding $u_{J}$.

On each spatial slice $\mathcal{M}(t)=\left\{x \in \mathbb{R}_{n}^{m+n} \mid(x, t) \in \mathcal{M}\right\}$, we take the metric induced from $\mathbb{R}_{n}^{m+n}$ and assume that it is spacelike. It will be convenient for us to use the following norm for the differential map $D u(x, t): \mathbb{R}^{m} \rightarrow \mathbb{R}^{n}$,

$$
\left\||D u \||(x, t)=\sup _{v \in \mathbb{R}^{m},|v|=1}|D u(x, t)(v)| .\right.
$$

Here $|\cdot|$ denotes the usual Euclidean norm, and $D$ is taken with respect to the space variables only (as usual). It is possible to show that $\|D u\|^{2}$ will be equal to the largest eigenvalue of $D u^{T} D u$ at each point, and that $|\|D u\|| \leq|D u| \leq \sqrt{m}\|D u\|$. Using the obvious relationship between $\|D u\|$ and the eigenvalues of the induced metric, we see that the graph will be spacelike if and only if $\|D u\|<1$. If $\Omega$ is convex and $\|D u\| \|^{2}<1-\kappa$ then it is easy to check that, for any $t \in I,{ }^{2}$

$$
|u(\hat{x}, t)-u(\hat{y}, t)| \leq \sup _{\Omega}|| D u(\cdot, t)|||| \hat{x}-\hat{y}\left|\leq(1-\kappa)^{1 / 2}\right| \hat{x}-\hat{y} \mid,
$$

and then, whenever $s \geq t$ are both in $I$,

$$
\begin{aligned}
|u(\hat{x}, t)-u(\hat{y}, s)| & \leq|u(\hat{x}, t)-u(\hat{y}, t)|+|u(\hat{y}, t)-u(\hat{y}, s)| \\
& \leq(1-\kappa)^{1 / 2}|\hat{x}-\hat{y}|+(s-t) \sup _{(t, s)}\left|\partial_{t} u(\hat{y}, \cdot)\right| .
\end{aligned}
$$

We denote by $H(x, t)$ the mean curvature vector at the point $x$ of the spatial slice $M(t)$. We will consider graphic flows that satisfy the quasilinear system

$$
\partial_{t} u=\hat{g}^{i j}(D u) \partial_{i j} u
$$

\footnotetext{
${ }^{2}$ To prove this, take $\hat{x} \in \Omega$ and let $h \in \mathbb{R}^{m}$ be such that $\hat{x}+\delta h \in \Omega$ for all $\delta \in[0,1]$. Then $|u(\hat{x}+h)-u(\hat{x})|=\left|\left(\int_{0}^{1} D u(\hat{x}+\delta h) d \delta\right) \cdot h\right| \leq \int_{0}^{1}|D u(\hat{x}+\delta h) \cdot h| d \delta=|h| \int_{0}^{1}|D u(\hat{x}+\delta h) \cdot h| /|h| d \delta \leq$ $|h| \sup _{\Omega}\||D u|\|$.
} 
on $\Omega \times I$, where $\hat{g}_{i j}=\delta_{i j}-\partial_{i} u^{v} \partial_{j} u^{v}$ gives the components of the induced metric on each spatial slice. This system will be parabolic because the spacelike condition implies that $\hat{g}=I-D u^{T} D u$ will be positive definite.

Proposition 1. Let $\mathcal{M}$ be a graph as in (2), and let I be open. Then $\mathcal{M}$ is a mean curvature flow in $\mathbb{R}_{n}^{m+n} \times \mathbb{R}$ if and only if the function $u$ satisfies the system (5).

Proof. If (5) holds then, to show that we have a mean curvature flow, it is enough to get parametrisations $F$ of our spatial slices with $\partial_{t} F=H$. In other words, for each $s \in I$, we hope to find $\phi$ such that $F(\hat{x}, t)=(\phi(\hat{x}, t), u(\phi(\hat{x}, t), t))$ satisfies $\partial_{t} F(\hat{x}, t)=H(F(\hat{x}, t), t)$ for times $t$ close to $s$. But we know that the mean curvature of our graph is $\left(0, \hat{g}^{i j} \partial_{i j} u\right)^{\perp}$, and that $\partial_{t} u=\hat{g}^{i j} \partial_{i j} u$. These facts and the chain rule applied to $F$ imply that we need $\partial_{t} F=\left(\partial_{t} \phi, D u \partial_{t} \phi\right)+\left(0, \partial_{t} u\right)$ to be equal to $\left(0, \partial_{t} u\right)^{\perp}$. This is equivalent to the system $\partial_{t} \phi^{j}=\left.\partial_{t} u \cdot \partial_{j} u \hat{g}^{i j}(D u)\right|_{(\phi(\hat{x}, t), t)}$ for $j=1, \ldots, m$. By thinking of $\hat{x}$ as being fixed, we can think of this as a system of ordinary differential equations and solve for some $\phi(t)$ with initial condition $\phi(s)=\hat{x}$, for any $\hat{x} \in \Omega$. By the usual existence and uniqueness theorems [Lee 2003, Theorem 17.15], solutions $\phi_{\hat{x}, s}(t)$ will exist for each $\hat{x} \in \Omega$ and $s \in I$. If we write $\phi_{\hat{x}, s}(t)=\phi_{s}(\hat{x}, t)$, then $\phi_{s}(\cdot, s)$ is the identity, $\phi_{s}$ is defined on some open set $\mathscr{E} \subset \Omega \times I$ containing $\Omega \times\{s\}$, and each $\phi_{s}(\cdot, t)$ will be a diffeomorphism [Lee 2003, Problem 17-15]. Then $\phi_{s}$ is the required function, so we have a mean curvature flow.

Conversely, if $\mathcal{M}$ is a mean curvature flow then we take $F=(\hat{F}, \tilde{F})$ such that $\partial_{t} F=H$ and $F(\hat{x}, t)=(\hat{F}(\hat{x}, t), u(\hat{F}(\hat{x}, t), t))$. By the chain rule, this gives $\partial_{t} F(\hat{x}, t)=(I, D u(\hat{F}(\hat{x}, t), t)) \cdot \partial_{t} \hat{F}(\hat{x}, t)+\left(0, \partial_{t} u(\hat{F}(\hat{x}, t), t)\right)$. The lefthand side is a normal vector and the first term on the right-hand side is tangential, therefore $\partial_{t} F(\hat{x}, t)=\left.\left(0, \partial_{t} u\right)^{\perp}\right|_{(\hat{F}(\hat{x}, t), t)}$. We already know that $\partial_{t} F(\hat{x}, t)=$ $H(F(\hat{x}, t), t)$, but the mean curvature at $F(\hat{x}, t)$ is given by $\left.\left(0, \hat{g}^{i j} \partial_{i j} u\right)^{\perp}\right|_{(\hat{F}(\hat{x}, t), t)}$. Hence $\left(0, \partial_{t} u\right)^{\perp}=\left(0, \hat{g}^{i j} \partial_{i j} u\right)^{\perp}$, and from here it is easy to check that we must have $\partial_{t} u=\hat{g}^{i j} \partial_{i j} u$.

Assumption 2. $M$ is a graphic flow, as in (2), where $\Omega$ is a convex, smooth domain in $\mathbb{R}^{m}$, and where the smooth function $u: \Omega \times I \rightarrow \mathbb{R}^{n}$ satisfies the system (5) and the inequality $\|D u\|^{2} \leq 1-\kappa$ for some constant $\kappa>0$.

For such flows, and for times $t$ on the interior of $I$, we can use the parametrisation $F$ from the proof of Proposition 1 to prove the following facts. Note that we will repeatedly use the fact that $\partial_{t} F=H=\Delta_{\mathcal{M}(t)} F$, by Equation (1), and we will write $g=\left(g_{i j}\right)=\left(\left\langle\partial_{i} F, \partial_{j} F\right\rangle\right)$ for the induced metric on spatial slices. The first fact is a version of the divergence theorem on mean curvature flows,

$$
\int_{\mathcal{M}(t)}\langle H, V\rangle=\int_{\mathcal{M}(t)}\left\langle\Delta_{M(t)} F, V\right\rangle=-\int_{\mathcal{M}(t)} \operatorname{div}_{\mathcal{M}(t)} V
$$


for vector fields $V$ with compact support on $\mu(t)$, where the integrals are taken over the spatial slice with respect to the induced metric $g .{ }^{3}$ If $f(x, t)$ is a real-valued function defined on the flow then

$$
\frac{d f}{d t}=\partial_{t} f+\langle\bar{g} D f, H\rangle \quad \text { and } \quad \Delta_{\mathcal{M}(t)} f=\langle H, \bar{g} D f\rangle+\operatorname{div}_{\mathcal{M}(t)}(\bar{g} D f),
$$

where $\bar{g}$ is the matrix defined in the previous section. The second equation here, along with the divergence theorem above, gives

$$
\int_{\mathcal{M}(t)}\left(\phi \Delta_{\mathcal{M}(t)} \eta-\eta \Delta_{\mathcal{M}(t)} \phi\right)=0
$$

whenever $\phi$ and $\eta$ are $C^{2}$ on $\mathcal{M}(t)$ with $\phi$ having compact support. Finally, using the usual formula for differentiating determinants, we have the following evolution equation on mean curvature flows,

$$
\frac{d}{d t} \sqrt{\operatorname{det} g}=-\sqrt{\operatorname{det} g}\langle H, H\rangle \geq 0 .
$$

Definition 3. Let $X_{0}=\left(x_{0}, t_{0}\right) \in \mathbb{R}^{m+n, 1}$. Define $\Phi_{X_{0}}: \mathbb{R}^{m+n} \times\left(-\infty, t_{0}\right) \rightarrow \mathbb{R}$ by

$$
\Phi_{X_{0}}(x, t)=\frac{1}{\left(4 \pi\left(t_{0}-t\right)\right)^{m / 2}} \exp \left(-\frac{\left\langle x-x_{0}, x-x_{0}\right\rangle}{4\left(t_{0}-t\right)}\right) .
$$

Let $M$ be a graphic flow in $\mathbb{R}_{n}^{m+n} \times \mathbb{R}$, as in (2). For times $t<t_{0}$, we define

$$
\Theta\left(M, X_{0}, t\right)=\int_{x \in \mathcal{M}(t)} \Phi_{X_{0}}(x, t) .
$$

We see that

$$
\frac{\partial \Phi_{X_{0}}}{\partial t}=\frac{m \Phi_{X_{0}}}{2\left(t_{0}-t\right)}-\frac{\left\langle x-x_{0}, x-x_{0}\right\rangle \Phi_{X_{0}}}{4\left(t_{0}-t\right)^{2}}, \bar{g} D \Phi_{X_{0}}=-\frac{\left(x-x_{0}\right) \Phi_{X_{0}}}{2\left(t_{0}-t\right)} .
$$

These equations, combined with (6), give

$$
\begin{aligned}
\left(\frac{d}{d t}+\Delta_{M(t)}\right) & \Phi_{X_{0}} \\
=\partial_{t} \Phi_{X_{0}}+ & 2\left\langle\bar{g} D \Phi_{X_{0}}, H\right\rangle+\operatorname{div}_{\mathcal{M}(t)}\left(\bar{g} D \Phi_{X_{0}}\right) \\
=\partial_{t} \Phi_{X_{0}}+ & \operatorname{div}_{\mathcal{M}(t)}\left(\bar{g} D \Phi_{X_{0}}\right)+\frac{\left\langle\left(\bar{g} D \Phi_{X_{0}}\right)^{\perp},\left(\bar{g} D \Phi_{X_{0}}\right)^{\perp}\right\rangle}{\Phi_{X_{0}}} \\
& -\left\langle H-\frac{\left(\bar{g} D \Phi_{X_{0}}\right)^{\perp}}{\Phi_{X_{0}}}, H-\frac{\left(\bar{g} D \Phi_{X_{0}}\right)^{\perp}}{\Phi_{X_{0}}}\right\rangle \Phi_{X_{0}}+\langle H, H\rangle \Phi_{X_{0}} .
\end{aligned}
$$

\footnotetext{
${ }^{3}$ Whenever it will not cause confusion, we will write integrals of the form $\int_{x \in \mathcal{M}(t)} f(x, t) d x$ as $\int_{\mathcal{M}(t)} f$ to save space. Such integrals are always taken relative to the induced metric from $\mathbb{R}_{n}^{m+n}$. Similarly, we write $\Delta_{\mathcal{M}(t)} f(x, t)$ as $\Delta_{\mathcal{M}(t)} f$ when the meaning is clear.
} 
But the first three terms on the right-hand side of this equation add up to 0 since, by (9),

$$
\begin{gathered}
\operatorname{div}_{\mathcal{M}(t)}\left(\bar{g} D \Phi_{X_{0}}\right)=\frac{-m \Phi_{X_{0}}}{2\left(t_{0}-t\right)}+\frac{\Phi_{X_{0}}}{4\left(t_{0}-t\right)^{2}}\left\langle\left(x-x_{0}\right)^{\top},\left(x-x_{0}\right)^{\top}\right\rangle, \\
\frac{\left\langle\left(\bar{g} D \Phi_{X_{0}}\right)^{\perp},\left(\bar{g} D \Phi_{X_{0}}\right)^{\perp}\right\rangle}{\Phi_{X_{0}}}=\frac{\Phi_{X_{0}}}{4\left(t_{0}-t\right)^{2}}\left\langle\left(x-x_{0}\right)^{\perp},\left(x-x_{0}\right)^{\perp}\right\rangle .
\end{gathered}
$$

Now we use this, and the evolution equation for $\sqrt{\operatorname{det} g}$, to differentiate the integral $\int_{x \in \mathcal{M}(t)} \Phi_{X_{0}}(x, t) \phi(x, t)$ when $\phi$ is some nonnegative $C^{2}$ function where each $\phi(\cdot, t)$ has compact support on $M(t)$.

$$
\begin{aligned}
\frac{d}{d t} \int_{\mathcal{M}(t)} & \Phi_{X_{0}} \phi \\
= & \int_{\mathcal{M}(t)}\left(\phi \frac{d \Phi_{X_{0}}}{d t}+\Phi_{X_{0}} \frac{d \phi}{d t}-\langle H, H\rangle \phi \Phi_{X_{0}}\right) \\
= & \int_{\mathcal{M}(t)} \Phi_{X_{0}}\left(\frac{d}{d t}-\Delta_{\mathcal{M}(t)}\right) \phi+\left(\left(\frac{d}{d t}+\Delta_{\mathcal{M}(t)}\right) \Phi_{X_{0}}-\langle H, H\rangle \Phi_{X_{0}}\right) \phi \\
= & \int_{\mathcal{M}(t)} \Phi_{X_{0}}\left(\frac{d}{d t}-\Delta_{\mathcal{M}(t)}\right) \phi-\left\langle H-\frac{\left(\bar{g} D \Phi_{X_{0}}\right)^{\perp}}{\Phi_{X_{0}}}, H-\frac{\left(\bar{g} D \Phi_{X_{0}}\right)^{\perp}}{\Phi_{X_{0}}}\right\rangle \phi \Phi_{X_{0}},
\end{aligned}
$$

where we have used (7) and then (10). By (9) this gives:

$$
\begin{aligned}
\frac{d}{d t} \int_{\mathcal{M}(t)} \Phi_{X_{0}} \phi=\int_{\mathcal{M}(t)} \Phi_{X_{0}} & \left(\frac{d}{d t}-\Delta_{\mathcal{M}(t)}\right) \phi \\
& -\int_{\mathcal{M}(t)}\left\langle H-\frac{\left(x-x_{0}\right)^{\perp}}{2\left(t_{0}-t\right)}, H-\frac{\left(x-x_{0}\right)^{\perp}}{2\left(t_{0}-t\right)}\right\rangle \phi \Phi_{X_{0}} .
\end{aligned}
$$

This will be very useful later, and it is our first step towards the proof of monotonicity formulas. It is important to remember that the second term on the righthand side is nonnegative (since the flow is spacelike, which means that normal vectors will be timelike or zero). This is unlike the Euclidean case, where the corresponding term would be nonpositive.

Proposition 4. Let $\mathcal{M}$ be as in Assumption 2, but with $\Omega=\mathbb{R}^{m}$ and $I=(-\infty, T]$ for $T>0$. If , for every point $(x, t)$ on the flow, we have

$$
H(x, t)=\frac{x^{\perp}}{2 t},
$$

then $\mathcal{M} \cap\{X \mid \tau(X) \leq 0\}$ is invariant under parabolic dilations.

Proof. The idea (as for a similar result in [Ilmanen 1997]) is to assume that there is some point $Y=(y, t)$ on $\left.\mathcal{M}^{\prime}=\mathcal{M} \cap X \mid \tau(X) \leq 0\right\}$, but not on $D_{\lambda} \mathcal{M}^{\prime}$ for some $\lambda$. Then we take a compactly supported $C^{2}$ function $\phi$ with $\phi(y)=1$ and $\phi=0$ on $D_{\lambda} M(t)$. 
The transformation formula for integrals gives $\int_{D_{\lambda} M(t)} \phi=\lambda^{m} \int_{\mathcal{M}\left(t / \lambda^{2}\right)} \phi(\lambda x)$. Then our evolution equation for $\sqrt{\operatorname{det} g}$ implies that

$$
\begin{aligned}
& \frac{d}{d \lambda} \int_{D_{\lambda} M(t)} \frac{\phi}{\lambda^{m}} \\
& \quad=\int_{x \in M\left(t / \lambda^{2}\right)}\left(\frac{2 t}{\lambda^{3}} \phi(\lambda x)\left\langle H, \frac{x^{\perp}}{2 t / \lambda^{2}}\right\rangle+D \phi(\lambda x) \cdot x-\frac{2 t}{\lambda^{2}} D \phi(\lambda x) \cdot H+\frac{m}{\lambda} \phi(\lambda x)\right),
\end{aligned}
$$

where we have used Equation (12) to get $H=x^{\perp} /\left(2 t / \lambda^{2}\right)$ on $M\left(t / \lambda^{2}\right)$, and the fact that $\partial_{t} F=H$. We can deal with the first term by using the divergence theorem (and the fact that $H$ is a normal vector) to get

$$
\int_{x \in \mathcal{M}\left(t / \lambda^{2}\right)}\left\langle H, \phi(\lambda x) x^{\perp}\right\rangle=-\int_{x \in \mathcal{M}\left(t / \lambda^{2}\right)} \operatorname{div}_{\mathcal{M}\left(t / \lambda^{2}\right)}(\phi(\lambda x) x),
$$

and by using the fact that $\operatorname{div}_{\mathcal{M}\left(t / \lambda^{2}\right)}(\phi(\lambda x) x)=m \phi(\lambda x)+\lambda D \phi(\lambda x) \cdot x^{\top}$. It follows that

$$
\frac{d}{d \lambda} \int_{D_{\lambda} M(t)} \phi=0
$$

so $\int_{D_{\lambda} \mu} \phi$ remains constant as $\lambda$ varies. The contradiction proves our claim.

Proposition 5. Let $X, Y \in \mathbb{R}^{m+n, 1}, s<\tau(Y)$ and $\lambda>0$; then

$$
\Theta\left(D_{\lambda}(\mathcal{M}-X), Y, s\right)=\Theta\left(M, X+D_{1 / \lambda} Y, \tau(X)+s / \lambda^{2}\right) .
$$

Proof. If $M$ is given by $u: \Omega \times I \rightarrow \mathbb{R}^{n}$ and if $X=(\hat{x}, \tilde{x}, t)$, then $D_{\lambda}(\mathcal{M}-X)$ is given by $u_{\lambda, X}(\cdot, \cdot)=\lambda\left(u\left(\cdot / \lambda+\hat{x}, \cdot / \lambda^{2}+t\right)-\tilde{x}\right)$ on $D_{\lambda}(\Omega \times I-(\hat{x}, t))$. Then the transformation rule for integrals gives the expected result.

\section{Monotonicity for entire flows}

Given a flow satisfying Assumption 2, we say that it is an entire flow if $\Omega=\mathbb{R}^{m}$ and $I=(-\infty, T]$ for some $T \in(-\infty, \infty]$. If $\mathcal{M}$ is such an entire flow, then $\Theta\left(\mathcal{M}, X_{0}, t\right)$ is finite at points $X_{0}=\left(x_{0}, t_{0}\right)=\left(\hat{x}_{0}, u\left(\hat{x}_{0}, t_{0}\right), t_{0}\right)$ on $\mathcal{M}$ for times $t<t_{0}$. To prove this, we use $\sqrt{\operatorname{det} \hat{g}}<1$ and the fact that inequality (4) gives a bound on the exponent in $\Phi_{X_{0}}$ on the flow,

$$
\begin{array}{r}
-\frac{\left\langle x-x_{0}, x-x_{0}\right\rangle}{4\left(t_{0}-t\right)} \leq \frac{-\kappa\left|\hat{x}-\hat{x}_{0}\right|^{2}}{4\left(t_{0}-t\right)}+\frac{(1-\kappa)^{1 / 2} \sup _{\left(t, t_{0}\right)}\left|\partial_{t} u\left(\hat{x}_{0}, \cdot\right)\right|\left|\hat{x}-\hat{x}_{0}\right|}{2} \\
+\frac{\left(t_{0}-t\right)^{2} \sup _{\left(t, t_{0}\right)}\left|\partial_{t} u\left(\hat{x}_{0}, \cdot\right)\right|^{2}}{4\left(t_{0}-t\right)} .
\end{array}
$$

Here we can use the fact that $u$ is smooth, so the time derivative in this inequality will be bounded on $\left(t, t_{0}\right)$ by some constant. Also, the fact that $t<t_{0}$ is fixed means that $t_{0}-t>0$ will be constant. This means that, for large $\left|\hat{x}-\hat{x}_{0}\right|$, the first term in 
the right-hand side of (13) will dominate. So we have a bound on $\Theta\left(\mathcal{M}, X_{0}, t\right)$ by some integral known to be finite, being given by the usual formula for Gaussian integrals, $\int_{\mathbb{R}^{m}} \exp \left(-A_{i j} y^{i} y^{j} / 2\right) d y=\sqrt{(2 \pi)^{m} / \operatorname{det}\left(A_{i j}\right)}$. (Here the matrix $A_{i j}$ is constant, symmetric and positive definite. Almost all of the bounds on integrals that we use will follow from this.)

The simplest example is a nonmoving plane, where each spatial slice is a spacelike plane (independent of time). Then $D u$ is constant and $\partial_{t} u=0$. Obviously this implies that $\left|u(\hat{x}, t)-u\left(\hat{x}_{0}, t_{0}\right)\right|^{2}=\left|D u \cdot\left(\hat{x}-\hat{x}_{0}\right)\right|^{2}=\left(\hat{x}-\hat{x}_{0}\right)^{T} D u^{T} D u\left(\hat{x}-\hat{x}_{0}\right)$, where we know that $\hat{g}=I-D u^{T} D u$. For any point $X_{0}=\left(\hat{x}_{0}, u\left(\hat{x}_{0}, t_{0}\right), t_{0}\right)$ on the flow, we then see that

$$
\Theta\left(M, X_{0}, t\right)=\int_{\mathbb{R}^{m}} \frac{1}{\left(4 \pi\left(t_{0}-t\right)\right)^{m / 2}} \exp \left(-\frac{\left(\hat{x}-\hat{x}_{0}\right)^{T} \hat{g}\left(\hat{x}-\hat{x}_{0}\right)}{4\left(t_{0}-t\right)}\right) \sqrt{\operatorname{det} \hat{g}} d \hat{x}=1,
$$

where we again use the Gaussian integral formula. Therefore $\Theta$ is equal to 1 on nonmoving planes.

Theorem 6. Let $\mathcal{M}$ be an entire flow satisfying Assumption 2, and let the mean curvature $H$ be uniformly bounded on $M$. Then

$$
\frac{d}{d t} \Theta\left(\mathcal{M}, X_{0}, t\right)=-\int_{x \in \mathcal{M}(t)}\left\langle H(x, t)+\frac{\left(x-x_{0}\right)^{\perp}}{2\left(t_{0}-t\right)}, H(x, t)+\frac{\left(x-x_{0}\right)^{\perp}}{2\left(t_{0}-t\right)}\right\rangle \Phi_{X_{0}}
$$

when $X_{0}=\left(x_{0}, t_{0}\right) \in \mathcal{M}$ and $t<t_{0}$.

This theorem gives us a monotonicity formula, similar to Huisken's, for entire spacelike mean curvature flows. It tells us that $\Theta$ will be nondecreasing with respect to the time variable on such flows (since the right-hand side in the formula is nonnegative, by the spacelike condition). This is different to the Euclidean case, where the Gaussian density ratio would be nonincreasing. The proof of this theorem should be compared to the one in [Ecker 2004, p. 55]. We could even weaken the assumption on $H$, but for now it is enough to assume that it is bounded.

Proof. For each $R>0$ we can choose (as in [Ecker 2004, proof of Theorem 4.13]) functions $\chi_{R}^{m}: \mathbb{R}^{m} \rightarrow \mathbb{R}$ such that $\chi_{B_{R}^{m}(0)} \leq \chi_{R}^{m} \leq \chi_{B_{2 R}^{m}(0)}$ and $R\left|D \chi_{R}^{m}\right|+R^{2}\left|D^{2} \chi_{R}^{m}\right| \leq$ $C$ for some constant $C$. ${ }^{4}$ Using these functions, we define $\chi_{R}: \mathbb{R}_{n}^{m+n} \rightarrow \mathbb{R}$ by taking $\chi_{R}(x)=\chi_{R}(\hat{x}, \tilde{x})=\chi_{R}^{m}(\hat{x})$ for any $x=(\hat{x}, \tilde{x})$. We apply (11) with $\phi=\chi_{R}$ to get

$$
\begin{aligned}
\frac{d}{d t} \int_{\mathcal{M}(t)} \Phi_{X_{0}} \chi_{R}=-\int_{\mathcal{M}(t)}\left\langle H+\frac{\left(x-x_{0}\right)^{\perp}}{2\left(t_{0}-t\right)},\right. & \left.H+\frac{\left(x-x_{0}\right)^{\perp}}{2\left(t_{0}-t\right)}\right\rangle \Phi_{X_{0}} \chi_{R} \\
& +\int_{\mathcal{M}(t)} \Phi_{X_{0}}\left(\frac{d}{d t}-\Delta_{\mathcal{M}(t)}\right) \chi_{R} .
\end{aligned}
$$

\footnotetext{
${ }^{4}$ For a set $K$, we denote by $\chi_{K}$ the characteristic function of $K$.
} 
Using (6), the Schwarz inequality and the bounds on the eigenvalues of $\hat{g}$ (from the assumed bound on the gradient), we have ${ }^{5}$

$$
\left|\left(\frac{d}{d t}-\Delta_{M(t)}\right) \chi_{R}\right| \leq\left|\hat{g}^{-1}(D u)\right| \cdot\left|D^{2} \chi_{R}^{m}\right| \leq C_{0}(\kappa) \frac{C}{R^{2}} \chi_{B_{2 R}^{m}(0)-B_{R}^{m}(0)},
$$

where we have also used the fact that $\chi_{R}^{m}$ is constant outside $B_{2 R}^{m}(0)-B_{R}^{m}(0)$.

Now we will restrict to any fixed bounded time interval $I^{\prime}=[a, b] \subset\left(-\infty, t_{0}\right)$, considering only times $t \in I^{\prime}$. The first thing to note here is that we have positive upper and lower bounds, independent of $t$ (but depending on $I^{\prime}$ ), on both $t_{0}-t$ and $1 /\left(t_{0}-t\right)$. Next we note that the flow is smooth on $\left(-\infty, t_{0}\right]$ (by our assumptions in the statement of the theorem) and $X_{0}=\left(\hat{x}_{0}, u\left(\hat{x}_{0}, t_{0}\right), t_{0}\right)$ lies on the flow, so we have $\sup _{\left[t, t_{0}\right]}\left|\partial_{t} u\left(\hat{x}_{0}, \cdot\right)\right| \leq \sup _{\left[a, t_{0}\right]}\left|\partial_{t} u\left(\hat{x}_{0}, \cdot\right)\right|$, where $\sup _{\left[a, t_{0}\right]}\left|\partial_{t} u\left(\hat{x}_{0}, \cdot\right)\right|$ is a finite constant independent of $t \in I^{\prime}$. We can use this to apply inequality (4) to bound the exponent of $\Phi_{X_{0}}$ on our flow, getting

$$
\begin{aligned}
-\frac{\left\langle x-x_{0}, x-x_{0}\right\rangle}{4\left(t_{0}-t\right)} \leq \frac{-\kappa\left|\hat{x}-\hat{x}_{0}\right|^{2}}{4\left(t_{0}-a\right)}+\frac{\left(t_{0}-a\right) \sup _{\left[a, t_{0}\right]}\left|\partial_{t} u\left(\hat{x}_{0}, \cdot\right)\right|^{2}}{4} & \\
& +\frac{(1-\kappa)^{1 / 2} \sup _{\left[a, t_{0}\right]}\left|\partial_{t} u\left(\hat{x}_{0}, \cdot\right)\right|}{2}\left|\hat{x}-\hat{x}_{0}\right| .
\end{aligned}
$$

We denote the right-hand side of this inequality by $Q\left(\left|\hat{x}-\hat{x}_{0}\right|\right)$, where the coefficients of the polynomial $Q$ depend on $I^{\prime}$ and $\hat{x}_{0}$ but are independent of $t \in I^{\prime}$. Also, using the Schwarz and triangle inequalities, with the assumed bounds on $H$ and $\|D u\| \|$ (and hence on the eigenvalues of $\hat{g}$ ), it is not difficult to see that we have $-\left\langle H+\left(x-x_{0}\right)^{\perp} / 2\left(t_{0}-t\right), H+\left(x-x_{0}\right)^{\perp} / 2\left(t_{0}-t\right)\right\rangle \leq P\left(\left|\hat{x}-\hat{x}_{0}\right|\right)$ on our flow, where $P$ is some polynomial with coefficients again independent of $t \in I^{\prime}$. Now we recall (14) and use it to get

$$
\begin{aligned}
& \left|\frac{d}{d t} \int_{\mathcal{M}(t)} \Phi_{X_{0}} \chi_{R}+\int_{\mathcal{M}(t)}\left\langle H+\frac{\left(x-x_{0}\right)^{\perp}}{2\left(t_{0}-t\right)}, H+\frac{\left(x-x_{0}\right)^{\perp}}{2\left(t_{0}-t\right)}\right\rangle \Phi_{X_{0}}\right| \\
& \leq\left|\int_{\mathcal{M}(t)} \Phi_{X_{0}}\left(\frac{d}{d t}-\Delta_{\mathcal{M}(t)}\right) \chi_{R}\right| \\
& \quad+\left|\int_{\mathcal{M}(t)}\left\langle H+\frac{\left(x-x_{0}\right)^{\perp}}{2\left(t_{0}-t\right)}, H+\frac{\left(x-x_{0}\right)^{\perp}}{2\left(t_{0}-t\right)}\right\rangle \Phi_{X_{0}}\left(1-\chi_{R}\right)\right| \\
& \leq \int_{\mathbb{R}^{m}} \frac{C_{0} C}{R^{2}} \frac{\chi_{B_{2 R}^{m}(0)-B_{R}^{m}(0)}^{\left(4 \pi\left(t_{0}-b\right)\right)^{m / 2}} \exp \left(Q\left(\left|\hat{x}-\hat{x}_{0}\right|\right)\right) d \hat{x}}{\quad+\int_{\mathbb{R}^{m}} P\left(\left|\hat{x}-\hat{x}_{0}\right|\right) \frac{\left(1-\chi_{B_{R}^{m}(0)}\right)}{\left(4 \pi\left(t_{0}-b\right)\right)^{m / 2}} \exp \left(Q\left(\left|\hat{x}-\hat{x}_{0}\right|\right)\right) d \hat{x},}
\end{aligned}
$$

${ }^{5}$ From now on, $C(\cdot, \ldots, \cdot)$ will always denote a positive constant depending on the quantities in parentheses. 
where we have used all of the inequalities above, as well as $\sqrt{\operatorname{det} \hat{g}} \leq 1$. Both integrands in the right-hand side are bounded by an integrable function independent of $R$ (since $Q$ is dominated by the $-\left|\hat{x}-\hat{x}_{0}\right|^{2}$ term and $P$ is just a polynomial). Both integrands converge pointwise to zero on $\mathbb{R}^{m}$ as $R \rightarrow \infty$, which allows us to apply the dominated convergence theorem to see that the right-hand side of this inequality converges to zero. Since the right-hand side is independent of $t \in I^{\prime}$, this convergence is uniform. So we have

$$
\lim _{R \rightarrow \infty} \frac{d}{d t} \int_{\mathcal{M}(t)} \Phi_{X_{0}} \chi_{R}=-\int_{\mathcal{M}(t)}\left\langle H+\frac{\left(x-x_{0}\right)^{\perp}}{2\left(t_{0}-t\right)}, H+\frac{\left(x-x_{0}\right)^{\perp}}{2\left(t_{0}-t\right)}\right\rangle \Phi_{X_{0}} .
$$

The uniform convergence allows us to swap the order of the limit and the derivative on the left-hand side to get

$$
-\int_{\mathcal{M}(t)}\left\langle H+\frac{\left(x-x_{0}\right)^{\perp}}{2\left(t_{0}-t\right)}, H+\frac{\left(x-x_{0}\right)^{\perp}}{2\left(t_{0}-t\right)}\right\rangle \Phi_{X_{0}}=\frac{d}{d t} \int_{\mathcal{M}(t)} \Phi_{X_{0}},
$$

where we have again used a dominated convergence argument (involving $Q$, etc.) and the fact that $\chi_{R}^{m}$ converges to 1 pointwise. Since we can do this for any such $I^{\prime}$, the equation above holds for all $t<t_{0}$.

Corollary 7. Let $\mathcal{M}$ be as in Theorem 6 , then $\Theta(\mathcal{M}, X, t) \leq 1$ for all $X \in \mathcal{M}$ and all $t<\tau(X)$. Also, $\Theta(M, X, t)=1$ for all $X \in \mathcal{M}$ and all $t<\tau(X)$ if and only if $M$ is a nonmoving plane.

Proof. Let $Y=(y, s) \in \mathcal{M}$, then we claim that $\lim _{t \rightarrow s} \Theta(\mathcal{M}, Y, t)=1$. We prove this by considering dilations of the flow using Proposition 5.

$$
\Theta(M, Y, t)=\Theta\left(D_{1 /(s-t)^{1 / 2}}(\mathcal{M}-Y), 0,-1\right)
$$

and, since the flow is smooth at $Y$, the flows $D_{1 /(s-t)^{1 / 2}}(\mathcal{M}-Y)$ converge to a nonmoving plane as $t \rightarrow s$. To understand why, write $\lambda=\sqrt{s-t}$ and let each $D_{1 / \lambda}(\mathcal{M}-Y)$ be given by the graph of a function $u_{\lambda}$. If $(\mathcal{M}-Y)$ is the graph of a function $u$, then $u_{\lambda}(\hat{z}, r)=u\left(\lambda \hat{z}, \lambda^{2} r\right) / \lambda$ and the definition of the derivative (with respect to $\lambda$ ) gives $\lim _{\lambda \rightarrow 0} u_{\lambda}(\hat{z}, r)=D u(0,0) \cdot \hat{z}+0 \cdot 2 r \partial_{t} u(0,0)$. Therefore $D_{1 / \lambda}(M-Y)$ converges pointwise to a nonmoving plane as $\lambda \rightarrow 0$.

We easily see that

$$
D u_{\lambda}(\hat{z}, r)=D u\left(\lambda \hat{z}, \lambda^{2} r\right) \rightarrow D u(0,0),
$$

so $\operatorname{det} \hat{g}\left(D u_{\lambda}\right)$ converges to $\operatorname{det} \hat{g}(D u(0,0))$. Also,

$$
\sup _{[-1,0]}\left|\partial_{t} u_{\lambda}(0, \cdot)\right|=\lambda \sup _{\left[-\lambda^{2}, 0\right]}\left|\partial_{t} u(0, \cdot)\right| \rightarrow 0
$$

as $\lambda \rightarrow 0$. We can use these facts to apply the dominated convergence theorem to $\Theta\left(D_{1 / \lambda}(\mathcal{M}-Y), 0,-1\right)$, by again using inequality (4) in the usual way to get an 
upper bound on the exponent of $\Phi_{0}(\cdot,-1)$ on each $D_{1 / \lambda}(M-Y)$,

$$
\begin{aligned}
\frac{-|\hat{x}|^{2}+\left|u_{\lambda}(\hat{x},-1)\right|^{2}}{4} & \leq \frac{-|\hat{x}|^{2}+\left((1-\kappa)^{1 / 2}|\hat{x}|+\sup _{[-1,0]}\left|\partial_{t} u_{\lambda}(0, \cdot)\right|\right)^{2}}{4} \\
& \leq \frac{-\kappa|\hat{x}|^{2}+2(1-\kappa)^{1 / 2}|\hat{x}|+1}{4},
\end{aligned}
$$

whenever $\lambda$ is small enough that $\sup _{[-1,0]}\left|\partial_{t} u_{\lambda}(0, \cdot)\right| \leq 1$. Now we have a bound (for all small $\lambda)$ on the integrands of each $\Theta\left(D_{1 / \lambda}(M-Y), 0,-1\right)$ by some integrable function. We can therefore apply the dominated convergence theorem to get $\Theta\left(D_{1 / \lambda}(\mathcal{M}-Y), 0,-1\right) \rightarrow 1$ as $\lambda \rightarrow 0$, since $\Theta$ is always equal to 1 on nonmoving planes. This fact and (16) give $\Theta(\mathcal{M}, Y, t) \rightarrow 1$ as $t \rightarrow s$. The monotonicity theorem tells us that $\Theta(M, Y, t)$ is nondecreasing with respect to $t<s$ and therefore must be $\leq 1$.

For the second part of the corollary, if $\Theta(M, Y, t) \equiv 1$ then the monotonicity formula gives

$$
0=\frac{d}{d t} \Theta(M, Y, t)=-\int_{\mathcal{M}(t)}\left\langle H+\frac{(x-y)^{\perp}}{2(s-t)}, H+\frac{(x-y)^{\perp}}{2(s-t)}\right\rangle \Phi_{Y},
$$

and therefore (since normal vectors are timelike or zero) we have

$$
H(x, t)=-(x-y)^{\perp} / 2(s-t) .
$$

This means that the flow $\mathcal{M}^{\prime}=(\mathcal{M}-Y) \cap\{X \mid \tau(X) \leq 0\}$ satisfies (12) and must be invariant under parabolic dilations. As $\lambda \rightarrow \infty$, the flows $D_{\lambda} \mathcal{M}^{\prime}$ again converge to a nonmoving plane, which must be equal to $M^{\prime}$. This is true for all $Y \in M$ with $\tau(T)<T=\sup I$, so $\mathcal{M}$ must be a nonmoving plane.

\section{Local monotonicity}

If a flow satisfying Assumption 2 has $I=[a, b)$, then Proposition 17 (given in the Appendix) implies that we can extend it continuously to $[a, b]$. Taking a subset of $\Omega$ if necessary (remember that we are interested in local theorems here), the following assumption will hold.

Assumption 8. With $M$ as in Assumption $2, \Omega \times I$ is bounded and $u$ is continuous on its closure.

Now we will prove a kind of local monotonicity theorem, which will be used to prove a local regularity theorem later. We will need to define a local version of $\Theta$. We can choose a $C^{2}$ function $\phi: \mathbb{R}^{m} \rightarrow \mathbb{R}$ which satisfies $\chi_{B_{1 / 2}^{m}(0)} \leq \phi \leq \chi_{B_{1}^{m}(0)}$ and $\left|D^{2} \phi\right| \leq C_{1}$, where $C_{1}$ is some positive constant depending only on $m$. Then, for any spacetime point $X_{0}=\left(\hat{x}_{0}, \tilde{x}_{0}, t_{0}\right)$ and any $\rho>0$, we define a function on 
$\mathbb{R}^{m+n}$ by

$$
\phi_{\rho, X_{0}}(x)=\phi_{\rho, X_{0}}(\hat{x}, \tilde{x})=\phi\left(\frac{\hat{x}-\hat{x}_{0}}{\rho}\right) \text {, }
$$

which will satisfy $\chi_{B_{\rho / 2}^{m}\left(\hat{x}_{0}\right) \times \mathbb{R}^{n}} \leq \phi_{\rho, X_{0}} \leq \chi_{B_{\rho}^{m}\left(\hat{x}_{0}\right) \times \mathbb{R}^{n}}$ and $\left|D^{2} \phi_{\rho, X_{0}}\right| \leq C_{1} / \rho^{2}$. It will also be convenient now for us to define the sets $Q_{\rho}^{m, n, 1}(X)=B_{\rho}^{m}(\hat{x}) \times \mathbb{R}^{n} \times\left(t-\rho^{2}, t\right)$ and $P_{\rho}^{m, n, 1}(X)=B_{\rho}^{m}(\hat{x}) \times \mathbb{R}^{n} \times\left(t-\rho^{2}, t+\rho^{2}\right)$ for any spacetime point $X=(\hat{x}, \tilde{x}, t)$.

Definition 9. Let $\mathcal{M}$ be a graphic flow in $\mathbb{R}_{n}^{m+n} \times \mathbb{R}$, as in (2). If $X_{0} \in \mathbb{R}^{m+n, 1}$ and $\rho>0$ are such that $Q_{\rho}^{m, n, 1}\left(X_{0}\right) \subset \Omega \times \mathbb{R}^{n} \times I$ then we define

$$
\Theta\left(M, X_{0}, t, \rho\right)=\int_{x \in \mathcal{M}(t)} \Phi_{X_{0}}(x, t) \phi_{\rho, X_{0}}(x)
$$

for $t<\tau\left(X_{0}\right)$ in $I$.

As in Proposition 5, we can prove

$$
\Theta\left(D_{\lambda}(\mathcal{M}-X), Y, t, \rho\right)=\Theta\left(\mathcal{M}, X+D_{1 / \lambda} Y, \tau(X)+t / \lambda^{2}, \rho / \lambda\right) .
$$

By the dominated convergence theorem, we easily see that $\Theta(\mathcal{M}, X, s, \rho)$ is continuous with respect to $X \in \mathcal{M}$. Now we can prove a local monotonicity theorem. We will use the notation $\bar{M}$ for the closure of $M$.

Theorem 10. Let $M$ satisfy Assumption 8 , and let $\rho>0$. Then there exist positive constants $C_{2}$ and $\delta<\rho^{2}$ such that, whenever $X_{0} \in \bar{M}$ is such that $Q_{\rho}^{m, n, 1}\left(X_{0}\right) \subset$ $\Omega \times \mathbb{R}^{n} \times I$, the function $t \mapsto \Theta\left(\mathcal{M}, X_{0}, t, \rho\right)+C_{2} t$ will be nondecreasing with respect to $t \in\left(\tau\left(X_{0}\right)-\delta, \tau\left(X_{0}\right)\right)$.

Note that $C_{2}$ and $\delta$ will be independent of such points $X_{0}$, but will depend on $\mu$ and $\rho$.

Proof. We know from (11) that

$$
\frac{d}{d t} \Theta\left(\mathcal{M}, X_{0}, t, \rho\right) \geq \int_{\mathcal{M}(t)} \Phi_{X_{0}}\left(\frac{d}{d t}-\Delta_{\mathcal{M}(t)}\right) \phi_{\rho, X_{0}} .
$$

As in the proof of Theorem 6 , it is easy to check that $\left|\left(d / d t-\Delta_{\mathcal{M}(t)}\right) \phi_{\rho, X_{0}}\right| \leq$ $C_{3} \chi_{B_{\rho}^{m}\left(\hat{x}_{0}\right) \times \mathbb{R}^{n}-B_{\rho / 2}^{m}\left(\hat{x}_{0}\right) \times \mathbb{R}^{n}}$, where $C_{3}=C_{3}(\kappa, \rho)$ is constant. Let $\hat{x}, \hat{y} \in \bar{\Omega}$ and $t<s$ in $\bar{I}$ be such that $\rho / 2<|\hat{x}-\hat{y}|<\rho$. Then, by (3) and the triangle inequality, we have

$$
\begin{aligned}
& -|\hat{x}-\hat{y}|^{2}+|u(\hat{x}, s)-u(\hat{y}, t)|^{2} \\
& \leq-\kappa|\hat{x}-\hat{y}|^{2}+2(1-\kappa)^{1 / 2}|\hat{x}-\hat{y} \| u(\hat{x}, s)-u(\hat{x}, t)|+|u(\hat{x}, s)-u(\hat{x}, t)|^{2} \\
& \leq-\kappa \rho^{2} / 4+2(1-\kappa)^{1 / 2} \rho|u(\hat{x}, s)-u(\hat{x}, t)|+|u(\hat{x}, s)-u(\hat{x}, t)|^{2} .
\end{aligned}
$$


But, by uniform continuity of $u$ (since it is continuous on the closure of $\Omega \times I$ ), we can take $\delta>0$ (not depending on $\hat{x}, \hat{y}, s, t)$ such that the right-hand side of (19) will be $\leq-\kappa \rho^{2} / 8$ whenever $|t-s|<\delta .{ }^{6}$ Taking $s=\tau\left(X_{0}\right)$ and combining the above inequalities with the fact that $\sqrt{\operatorname{det} \hat{g}} \leq 1$ gives

$$
\left|\int_{\mathcal{M}(t)} \Phi_{X_{0}}\left(\frac{d}{d t}-\Delta_{\mathcal{M}(t)}\right) \phi_{\rho, X_{0}}\right| \leq \int_{\Omega} \frac{C_{3} \chi_{B_{\rho}^{m}\left(\hat{x}_{0}\right)-B_{\rho / 2}^{m}\left(\hat{x}_{0}\right)}}{\left(4 \pi\left(\tau\left(X_{0}\right)-t\right)\right)^{m / 2}} \exp \left(\frac{-\rho^{2} \kappa / 32}{\tau\left(X_{0}\right)-t}\right),
$$

for $0<\tau\left(X_{0}\right)-t<\delta$. Taking $t \rightarrow \tau\left(X_{0}\right)$ in the right-hand side shows that it is bounded by some finite constant $C_{4}$ for these values of $t$. Therefore

$$
\frac{d}{d t} \Theta\left(\mu, X_{0}, t, \rho\right) \geq-C_{4}
$$

for $t \in\left(\tau\left(X_{0}\right)-\delta, \tau\left(X_{0}\right)\right)$, proving the theorem.

Corollary 11. Let $\mathcal{M}$ be as in Theorem 10. If $X_{0} \in \bar{M}$ and $\rho_{0}>0$ are such that $Q_{\rho_{0}}^{m, n, 1}(Y) \subset \Omega \times \mathbb{R}^{n} \times I$ for all $Y \in Q_{\rho_{0}}^{m, n, 1}\left(X_{0}\right)$, and if

$$
\lim _{t \rightarrow \tau\left(X_{0}\right)} \Theta\left(\mathcal{M}, X_{0}, t, \rho_{0}\right)>1-\epsilon
$$

for some $\epsilon>0$, then there exists $\rho \in\left(0, \rho_{0}\right)$ such that

$$
\Theta\left(M, Y, t, \rho_{0}\right) \geq 1-\epsilon
$$

for all $Y \in Q_{\rho}^{m, n, 1}\left(X_{0}\right) \cap \mathcal{M}$ and all $t \in\left(\tau(Y)-\rho^{2}, \tau(Y)\right)$.

Proof. Let $\lim _{t \rightarrow \tau\left(X_{0}\right)} \Theta\left(\mathcal{M}, X_{0}, t, \rho_{0}\right) \geq 1-\epsilon+\eta$ for some $\eta>0$ (the limit exists in $\mathbb{R} \cup\{\infty\}$ by the local monotonicity theorem). Then there must exist $\rho_{1} \in\left(0, \rho_{0}\right]$ such that $\Theta\left(\mathcal{M}, X_{0}, \tau\left(X_{0}\right)-\rho_{1}^{2}, \rho_{0}\right)>1-\epsilon+\eta / 2$. We can choose $\rho_{1}$ to be as small as we like, so we take $\rho_{1}^{2}$ to be less than both $\delta\left(\mathcal{M}, \rho_{0}\right)$ and $\eta / 4 C_{2}\left(\mathcal{M}, \rho_{0}\right)$ (with $\delta$ and $C_{2}$ as in Theorem 10). By continuity, there will exist $\rho \in\left(0, \rho_{1}\right)$ such that, for all $Y \in Q_{\rho}^{m, n, 1}\left(X_{0}\right) \cap \mathcal{M}$,

$$
\Theta\left(M, Y, \tau\left(X_{0}\right)-\rho_{1}^{2}, \rho_{0}\right)>1-\epsilon+\eta / 4
$$

and $\left(\tau(Y)-\rho^{2}, \tau(Y)\right) \subset\left(\tau\left(X_{0}\right)-\rho_{1}^{2}, \tau\left(X_{0}\right)\right) \subset\left(\tau\left(X_{0}\right)-\delta, \tau\left(X_{0}\right)\right)$. So we can apply Theorem 10 to $\Theta\left(\mathcal{M}, Y, t, \rho_{0}\right)$ for $t \in\left(\tau(Y)-\rho^{2}, \tau(Y)\right)$ to get

$$
\Theta\left(\mathcal{M}, Y, \tau\left(X_{0}\right)-\rho_{1}^{2}, \rho_{0}\right)+C_{2}\left(\tau\left(X_{0}\right)-\rho_{1}^{2}\right) \leq \Theta\left(\mathcal{M}, Y, t, \rho_{0}\right)+C_{2} t
$$

for all such $Y$ and $t$, which in turn implies

$$
\Theta\left(M, Y, t, \rho_{0}\right) \geq C_{2}\left(\tau\left(X_{0}\right)-t-\rho_{1}^{2}\right)+1-\epsilon+\eta / 4 \geq 1-\epsilon+\left(\eta / 4-\rho_{1}^{2} C_{2}\right),
$$

where the last term is positive by our choice of $\rho_{1}$.

${ }^{6}$ Uniform continuity implies that, for any $\epsilon>0$, there exists $\delta>0$ such that $\|(\hat{x}, s)-(\hat{y}, t)\|<\delta$ implies $|u(\hat{x}, s)-u(\hat{y}, t)|<\epsilon$. Taking $\hat{x}=\hat{y}$ and a small enough $\epsilon$ here proves our claim. 
Proposition 12. Let $M$ satisfy Assumption 8 , and let $X_{0}$ and $\rho$ be as in Theorem 10. Then $\lim _{t \rightarrow \tau\left(X_{0}\right)} \Theta\left(\mathcal{M}, X_{0}, t, \rho\right)=\lim _{t \rightarrow \tau\left(X_{0}\right)} \Theta\left(M, X_{0}, t\right)$. In particular, the limit on the left-hand side is independent of $\rho$.

Proof. It is easy to see that, if we write $X_{0}=\left(\hat{x}_{0}, u\left(\hat{x}_{0}, t_{0}\right), t_{0}\right)$,

$$
\begin{aligned}
0 & \leq \Theta\left(M, X_{0}, t\right)-\Theta\left(M, X_{0}, t, \rho\right) \\
& =\int_{\Omega} \frac{\exp \left(\frac{-\left|\hat{x}-\hat{x}_{0}\right|^{2}+\left|u(\hat{x}, t)-u\left(\hat{x}_{0}, t_{0}\right)\right|^{2}}{4\left(t_{0}-t\right)}\right)}{\left(4 \pi\left(t_{0}-t\right)\right)^{m / 2}}\left(1-\phi\left(\frac{\hat{x}-\hat{x}_{0}}{\rho}\right)\right) \sqrt{\operatorname{det} \hat{g}} d \hat{x} .
\end{aligned}
$$

But $\sqrt{\operatorname{det} \hat{g}}<1$ and $1-\phi\left(\left(\hat{x}-\hat{x}_{0}\right) / \rho\right)$ is at most 1 and vanishes for $\hat{x} \in B_{\rho / 2}^{m}\left(\hat{x}_{0}\right)$. Thus we only need to consider $\left|\hat{x}-\hat{x}_{0}\right| \geq \rho / 2$ and, as in inequality (19), we get

$$
\begin{aligned}
& -\left|\hat{x}-\hat{x}_{0}\right|^{2}+\left|u(\hat{x}, t)-u\left(\hat{x}_{0}, t_{0}\right)\right|^{2} \\
& \quad \leq-\kappa\left|\hat{x}-\hat{x}_{0}\right|+2(1-\kappa)^{1 / 2}\left|\hat{x}-\hat{x}_{0}\right|\left|u\left(\hat{x}_{0}, t\right)-u\left(\hat{x}_{0}, t_{0}\right)\right|+\left|u\left(\hat{x}_{0}, t\right)-u\left(\hat{x}_{0}, t_{0}\right)\right|^{2} \\
& \quad \leq-\kappa \rho^{2} / 4+2(1-\kappa)^{1 / 2} \operatorname{diam} \Omega\left|u\left(\hat{x}_{0}, t\right)-u\left(\hat{x}_{0}, t_{0}\right)\right|+\left|u\left(\hat{x}_{0}, t\right)-u\left(\hat{x}_{0}, t_{0}\right)\right|^{2},
\end{aligned}
$$

which is $\leq-\kappa \rho^{2} / 8$ when we take $\left|u\left(\hat{x}_{0}, t\right)-u\left(\hat{x}_{0}, t_{0}\right)\right|$ small enough (by continuity) by taking $t$ close enough to $t_{0}$. Therefore, for such $t$, we have

$$
\Theta\left(M, X_{0}, t\right)-\Theta\left(M, X_{0}, t, \rho\right) \leq \int_{\Omega} \frac{\exp \left(\left(-\kappa \rho^{2} / 8\right) / 4\left(t_{0}-t\right)\right)}{\left(4 \pi\left(t_{0}-t\right)\right)^{m / 2}} d \hat{x}
$$

which converges to 0 as $t \rightarrow t_{0}$.

\section{Local regularity}

In [White 2005], a regularity theorem for mean curvature flows in Euclidean spaces is proved. To do this, a kind of local $C^{2, \alpha}$ norm is used (defined at each point of a flow and denoted by $K_{2, \alpha}$ ). For a sequence of $C^{2, \alpha}$ flows, denoted by $\mu_{J}$, if this norm is uniformly bounded on compact subsets as $J \rightarrow \infty$ then a version of the Arzelà-Ascoli theorem [White 2005, Theorem 2.6] gives local parabolic $C^{2}$ convergence of a subsequence to some locally $C^{2, \alpha}$ flow. However, the definition of this norm involves rotations, which would cause problems in the semi-Euclidean case (because of the spacelike condition and because the mean curvature flow system is not preserved by such rotations). It is convenient for us to define a slightly different quantity with similar properties. The idea will be to use the gradient bound (from the spacelike assumption) to ignore the first few terms in the $C^{2, \alpha}$ norm, thus removing the need to translate and rotate in the definition of $K_{2, \alpha}$.

Suppose that we have a spacelike, graphic flow $\mathcal{M}$ (as in (2), not necessarily a mean curvature flow) and $X \in \Omega \times \mathbb{R}^{n} \times I$. For any $\alpha \in(0,1)$, we define $G_{2, \alpha}(\mathcal{M}, X)$ 
to be the infimum of the numbers $\lambda>0$ such that

$$
\left[\left.D u_{\lambda, X}\right|_{U^{m, 1}}\right]_{\alpha}+\left\|\left.D^{2} u_{\lambda, X}\right|_{U^{m, 1}}\right\|_{0, \alpha}+\left\|\left.\partial_{t} u_{\lambda, X}\right|_{U^{m, 1}}\right\|_{0, \alpha} \leq 1
$$

where $u_{\lambda, X}$ is the function whose graph gives the flow $D_{\lambda}(\mu-X)$, and $\left.u_{\lambda, X}\right|_{U^{m, 1}}$ is the restriction to $U^{m, 1}=B_{1}^{m}(0) \times(-1,0]$. This will be finite when the flow is smooth (to understand why, see how each term in (20) is affected by dilations). It is important to note that, for any $X=(\hat{x}, \tilde{x}, t), G_{2, \alpha}(\mathcal{M}, X)$ is independent of $\tilde{x}$ (since the definition only involves derivatives of $u$ ). We will also need the obvious facts that this quantity will be zero on nonmoving planes and that $G_{2, \alpha}\left(D_{\lambda}(\mathcal{M}-X), 0\right)=$ $G_{2, \alpha}(\mathcal{M}, X) / \lambda$.

The most important property of $G_{2, \alpha}$ is a version of the Arzelà-Ascoli theorem. Roughly, if we have a sequence of smooth spacelike flows $M_{J}$, each containing the origin and with $G_{2, \alpha}\left(M_{J}, \cdot\right)$ uniformly bounded on compact subsets of spacetime as $J \rightarrow \infty$, then we have local parabolic $C^{2}$ convergence of some subsequence to a locally $C^{2, \alpha}$ limit flow. Comparing $G_{2, \alpha}$ to $K_{2, \alpha}$ and applying Theorem 2.6 of [White 2005] gives us this fact, but we will still explain in detail in Proposition 19 in a special case (the only case that we need). Furthermore, if each of the flows satisfies the system (5) then so will the limit (by the $C^{2}$ convergence). This limit must then be smooth by induction, since a $C^{k, \alpha}$ solution to the system must be $C^{k+1, \alpha}$, by the usual theorems for linear equations; see [Friedman 1964, Chapter $3]$, for example.

Theorem 13. Let $\alpha, \kappa \in(0,1)$ be given. Then there exist positive constants $\epsilon$ and $C_{5}$ such that if

(a) $M$ is as in Assumption 2, with $\sup I=0 \in I$ and with $u(0,0)=0$, and

(b) $\rho_{0}>1$ is such that $Q_{\rho_{0}}^{m, n, 1}(Y) \subset \Omega \times \mathbb{R}^{n} \times I$ and

$$
\Theta\left(\mathcal{M}, Y, t, \rho_{0}\right) \geq 1-\epsilon
$$

for all $Y \in Q_{1}^{m, n, 1}(0) \cap M$ and all $t \in(\tau(Y)-1, \tau(Y))$,

then $\sup _{X \in Q_{1}^{m, n, 1}(0)} G_{2, \alpha}(\mathcal{M}, X) d\left(X, P_{1}^{m, n, 1}(0)\right) \leq C_{5}$.

It is important to notice that the constants $\epsilon$ and $C_{5}$ will depend on $\kappa, \alpha, m, n$, but will be independent of $\mathcal{M}$. Also, since $G_{2, \alpha}$ scales like the reciprocal of parabolic distance, the inequality in the conclusion of the theorem is invariant under parabolic dilations. This is the most important theorem of this section and is a version of White's local regularity theorem. The proof should be compared to those of [White 2005, Theorem 3.1] and [Ecker 2004, Theorem 5.6]. As in this latter reference, we use the local version of $\Theta$. As in [White 2005], we aim for bounds on the $C^{2, \alpha}$ 
norm and use the Schauder estimates, ${ }^{7}$ rather than aiming for bounds on the second fundamental form and using related interior estimates as in [Ecker 2004].

Proof. Let $\bar{\epsilon}$ be the infimum of numbers $\epsilon>0$ for which the theorem fails (i.e., for which no such $C_{5}$ exists). We need $\bar{\epsilon}>0$, so we assume $\bar{\epsilon}=0$ to get a contradiction. We take a sequence $\epsilon_{J} \rightarrow \bar{\epsilon}$ with $\epsilon_{J}>\bar{\epsilon}$. Then there exist sequences $M_{J}$ and $\rho_{J}>1$, satisfying all of the assumptions of the theorem (with the same $\alpha$ and $\kappa$ ), but with $\epsilon_{J}, M_{J}, \rho_{J}$ in place of $\epsilon, \mathcal{M}, \rho_{0}$, and with

$$
\gamma_{J}=\sup _{X \in Q_{1}^{m, n, 1}(0)} d\left(X, P_{1}^{m, n, 1}(0)\right) G_{2, \alpha}\left(M_{J}, X\right) \rightarrow \infty
$$

as $J \rightarrow \infty$. Each $\gamma_{J}$ is finite since $\mu_{J}$ is smooth. For each $J$ we can choose $Y_{J}$ in $Q_{1}^{m, n, 1}(0)$ such that $G_{2, \alpha}\left(M_{J}, Y_{J}\right) d\left(Y_{J}, P_{1}^{m, n, 1}(0)\right) \geq \gamma_{J} / 2$, and we can assume that $Y_{J} \in \mathcal{M}_{J}{ }^{8}$ We define $\lambda_{J}=G_{2, \alpha}\left(\mathcal{M}_{J}, Y_{J}\right)$ and consider the flows ${ }^{9}$

$$
\tilde{M}_{J}=D_{\lambda_{J}}\left(\mu_{J}-Y_{J}\right)
$$

which all contain the origin (in spacetime). Then $G_{2, \alpha}\left(\tilde{M}_{J}, 0\right)=1$ for all $J$ and $D_{\lambda_{J}}\left(P_{1}^{m, n, 1}(0)-Y_{J}\right)=P_{\lambda_{J}}^{m, n, 1}\left(-D_{\lambda_{J}} Y_{J}\right)$. But now

$$
\frac{\gamma_{J}}{2} \leq G_{2, \alpha}\left(\mu_{J}, Y_{J}\right) d\left(Y_{J}, P_{1}^{m, n, 1}(0)\right)=1 \times d\left(0, P_{\lambda_{J}}^{m, n, 1}\left(-D_{\lambda_{J}} Y_{J}\right)\right)
$$

so $d\left(0, P_{\lambda_{J}}^{m, n, 1}\left(-D_{\lambda_{J}} Y_{J}\right)\right) \rightarrow \infty$ since $\gamma_{J} \rightarrow \infty$ as $J \rightarrow \infty$. Let $X$ be a point in $Q_{\lambda_{J}}^{m, n, 1}\left(-D_{\lambda_{J}} Y_{J}\right)$. Then

$$
d\left(X, P_{\lambda_{J}}^{m, n, 1}\left(-D_{\lambda_{J}} Y_{J}\right)\right) G_{2, \alpha}\left(\tilde{M}_{J}, X\right) \leq \gamma_{J} \leq 2 d\left(0, P_{\lambda_{J}}^{m, n, 1}\left(-D_{\lambda_{J}} Y_{J}\right)\right),
$$

from which we obtain

$$
G_{2, \alpha}\left(\tilde{M}_{J}, X\right) \leq \frac{2 d\left(0, P_{\lambda_{J}}^{m, n, 1}\left(-D_{\lambda_{J}} Y_{J}\right)\right)}{d\left(X, P_{\lambda_{J}}^{m, n, 1}\left(-D_{\lambda_{J}} Y_{J}\right)\right)} .
$$

The triangle inequality gives $\|0-Y\| \leq\|0-X\|+\|Y-X\|$, and taking the supremum over all $Y \notin P_{\lambda_{J}}^{m, n, 1}\left(-D_{\lambda_{J}} Y_{J}\right)$ gives

$$
d\left(X, P_{\lambda_{J}}^{m, n, 1}\left(-D_{\lambda_{J}} Y_{J}\right)\right) \geq d\left(0, P_{\lambda_{J}}^{m, n, 1}\left(-D_{\lambda_{J}} Y_{J}\right)\right)-\|X\|
$$

\footnotetext{
${ }^{7}$ Note that White uses the Schauder estimates for the heat equation but, since we do not want to rotate our flows, we have to use a more general version of the Schauder estimates.

${ }^{8}$ Remember that $G_{2, \alpha}(\mathcal{M},(\hat{x}, \tilde{x}, t))$ is independent of $\tilde{x}$, and so is $d\left((\hat{x}, \tilde{x}, t), P_{1}^{m, n, 1}(0)\right)$.

${ }^{9}$ Note that the flows $\mu_{J}$ and $\tilde{M}_{J}$ will be graphs of functions $u_{J}$ and $\tilde{u}_{J}$ on sets $\Omega_{J} \times I_{J}$ and $\tilde{\Omega}_{J} \times \tilde{I}_{J}$ respectively, where $\sup I_{J}=0 \Rightarrow \sup \tilde{I}_{J}=\tau\left(-D_{\lambda_{J}} Y_{J}\right)>0$.
} 
which leads to

$$
G_{2, \alpha}\left(\tilde{M}_{J}, X\right) \leq \frac{2}{1-\|X\| / d\left(0, P_{\lambda_{J}}^{m, n, 1}\left(-D_{\lambda_{J}} Y_{J}\right)\right)},
$$

whenever the right-hand side is positive. Since $d\left(0, P_{\lambda_{J}}^{m, n, 1}\left(-D_{\lambda_{J}} Y_{J}\right)\right) \rightarrow \infty$, this inequality tells us that $G_{2, \alpha}\left(\tilde{M}_{J}, X\right)$ is uniformly bounded (as $J \rightarrow \infty$ ) on compact subsets of spacetime with $\tau(X) \leq 0 .{ }^{10}$ This allows us to apply Proposition 19 to the sequence $\tilde{M}_{J} \cap\{X \mid \tau(X) \leq 0\}$ to get parabolic $C^{2}$ convergence, on compact subsets of $\mathbb{R}^{m} \times(-\infty, 0]$, of a subsequence to a limit flow $\mathcal{M}^{\prime}$. We can assume that this subsequence is our original sequence, and will therefore continue to use the notation $\tilde{M}_{J}$. The limit $\mathcal{M}^{\prime}$ will be a smooth entire graphic flow defined on $\mathbb{R}^{m} \times(-\infty, 0]$ (since $\left.\lambda_{J} \rightarrow \infty\right)$. It will be the graph of a function $u^{\prime}$ satisfying the system (5) (since the convergence is $C^{2}$ ). Also, since the gradient bound is unaffected by parabolic dilations, $\sup \left\|D u^{\prime}\right\|^{2} \leq 1-\kappa$. Proposition 19 tells us that $\mathcal{M}^{\prime}$ has uniformly bounded mean curvature. This allows us to apply the monotonicity theorem and related results to the flow.

Now we use the assumption that $\Theta\left(\mathcal{M}_{J}, Y, s, \rho_{J}\right) \geq 1-\epsilon_{J}$ for $Y \in Q_{1}^{m, n, 1}(0) \cap \mathcal{M}_{J}$ and $s \in(\tau(Y)-1, \tau(Y))$. By (17), this is equivalent to the inequality

$$
\Theta\left(\tilde{M}_{J}, Y, s, \lambda_{J} \rho_{J}\right) \geq 1-\epsilon_{J}
$$

for $Y \in Q_{\lambda_{J}}^{m, n, 1}\left(-D_{\lambda_{J}} Y_{J}\right) \cap \tilde{M}_{J}$ and $s \in\left(\tau(Y)-\lambda_{J}^{2}, \tau(Y)\right)$. Given $Z=\left(\hat{z}, u^{\prime}(\hat{z}, t), t\right)$ in $\mathcal{M}^{\prime}$, with $s<t<0$, we can take a sequence $Z_{J}=\left(\hat{z}, \tilde{u}_{J}(\hat{z}, t), t\right) \in \tilde{M}_{J}$ with $Z_{J} \rightarrow Z$. Then, for large enough $J$, the fact that $d\left(0, P_{\lambda_{J}}^{m, n, 1}\left(-D_{\lambda_{J}} Y_{J}\right)\right) \rightarrow \infty$ implies that $Z_{J}$ (which is bounded since it converges) will be in $Q_{\lambda_{J}}^{m, n, 1}\left(-D_{\lambda_{J}} Y_{J}\right)$. Obviously we will have $s \in\left(\tau\left(Z_{J}\right)-\lambda_{J}^{2}, \tau\left(Z_{J}\right)\right)$ for all large $J$. This gives $\Theta\left(\tilde{M}_{J}, Z_{J}, s, \lambda_{J} \rho_{J}\right) \geq 1-\epsilon_{J}$. We see easily that $\Theta\left(\tilde{M}_{J}, Z_{J}, s, \lambda_{J} \rho_{J}\right)$ equals

$$
\int_{\tilde{\Omega}_{J}} \frac{\exp \left(\frac{-|\hat{x}-\hat{z}|^{2}+\left|\tilde{u}_{J}(\hat{x}, s)-\tilde{u}_{J}(\hat{z}, t)\right|^{2}}{4(t-s)}\right)}{(4 \pi(t-s))^{m / 2}} \phi\left(\frac{\hat{x}-\hat{z}}{\lambda_{J} \rho_{J}}\right) \sqrt{\operatorname{det} \hat{g}\left(D \tilde{u}_{J}(\hat{x}, s)\right)} d \hat{x},
$$

where the integral can be thought of as an integral over $\mathbb{R}^{m}$ since $\phi$ has compact support. By the $C^{2}$ convergence $\tilde{u}_{J} \rightarrow u^{\prime}$ and the fact that $\rho_{J} \lambda_{J} \rightarrow \infty$ with $\phi \equiv 1$ in some ball with centre 0 , the integrands above will converge pointwise to the integrand in $\Theta\left(\mathcal{M}^{\prime}, Z, s\right)$. But we have $\phi \leq 1, \sqrt{\operatorname{det} \hat{g}} \leq 1$ and $t-s>0$ independent

\footnotetext{
${ }^{10}$ For example, for any such compact set we can assume $G_{2, \alpha}\left(\tilde{\mathcal{M}}_{J}, X\right) \leq 4$ for all $X$ in this set by assuming $\|X\| \leq R$ and taking $J$ so large that $d\left(0, P_{\lambda_{J}}^{m, n, 1}\left(-D_{\lambda_{J}} Y_{J}\right)\right) \geq 2 R$.
} 
of $J$, as well as

$$
\begin{aligned}
-|\hat{x}-\hat{z}|^{2}+\left|\tilde{u}_{J}(\hat{x}, s)-\tilde{u}_{J}(\hat{z}, t)\right|^{2} \leq & -\kappa|\hat{x}-\hat{z}|^{2}+(t-s)^{2} \sup _{(s, t)}\left|\partial_{t} \tilde{u}_{J}(\hat{z}, \cdot)\right|^{2} \\
& +2(1-\kappa)^{1 / 2}|\hat{x}-\hat{z}|(t-s) \sup _{(s, t)}\left|\partial_{t} \tilde{u}_{J}(\hat{z}, \cdot)\right|
\end{aligned}
$$

by inequality (4). By the parabolic $C^{2}$ convergence, we can assume for large $J$ that $\sup _{(s, t)}\left|\partial_{t} \tilde{u}_{J}(\hat{z}, \cdot)\right|$ is arbitrarily close to $\sup _{(s, t)}\left|\partial_{t} u^{\prime}(\hat{z}, \cdot)\right|$, which is finite (by smoothness of $u^{\prime}$ ) and independent of $J$. These inequalities combine to give a bound on the integrands of (22) by some function that is independent of $J$ and integrable over $\mathbb{R}^{m}$. This allows us to apply the dominated convergence theorem to get $\Theta\left(\mathcal{M}^{\prime}, Z, s\right) \leftarrow \Theta\left(\tilde{\mathcal{M}}_{J}, Z_{J}, s, \lambda_{J} \rho_{J}\right) \geq 1-\epsilon_{J} \rightarrow 1-\bar{\epsilon}$. So, for all $Z \in \mathcal{M}^{\prime}$ with $s<\tau(Z)<0$, we have $\Theta\left(\mathcal{M}^{\prime}, Z, s\right) \geq 1-\bar{\epsilon}$. Now, since we assumed $\bar{\epsilon}=0$, the fact that $\mathcal{M}^{\prime}$ is entire with $\Theta\left(\mathcal{M}^{\prime}, Z, s\right) \geq 1$ implies by Corollary 7 that $\Theta\left(\mathcal{M}^{\prime}, Z, s\right) \equiv 1$. Therefore $\mathcal{M}^{\prime}$ must be a nonmoving plane.

Let $u^{\prime}$ be as above and consider the linear operator with constant coefficients $\partial_{t}-\hat{g}^{i j}\left(D u^{\prime}\right) \partial_{i j}$ applied to $\tilde{u}_{J}$. The system (5) and the fact that $\partial_{i j} u^{\prime}=\partial_{t} u^{\prime}=0$ then give $\left(\partial_{t}-\hat{g}^{i j}\left(D u^{\prime}\right) \partial_{i j}\right)\left(\tilde{u}_{J}-u^{\prime}\right)=\left(\hat{g}^{i j}\left(D \tilde{u}_{J}\right)-\hat{g}^{i j}\left(D u^{\prime}\right)\right) \partial_{i j} \tilde{u}_{J}$. For $U_{2}^{m, 1}(0) \subset$ $\mathbb{R}^{m} \times(-\infty, 0]$, the Schauder estimates for linear parabolic equations [Krylov 1996, Theorem 8.11.1] tell us that

$$
\begin{aligned}
& \left\|\left.\left(\tilde{u}_{J}-u^{\prime}\right)\right|_{U_{2}^{m, 1}(0)}\right\|_{2, \alpha} \\
& \quad \leq C_{6}\left(\left\|\left.\left(\partial_{t}-\hat{g}^{i j}\left(D u^{\prime}\right) \partial_{i j}\right)\left(\tilde{u}_{J}-u^{\prime}\right)\right|_{U_{4}^{m, 1}(0)}\right\|_{0, \alpha}+\sup _{U_{4}^{m, 1}(0)}\left|\tilde{u}_{J}-u^{\prime}\right|\right) \\
& \quad=C_{6}\left(\left\|\left.\left(\hat{g}^{i j}\left(D \tilde{u}_{J}\right)-\hat{g}^{i j}\left(D u^{\prime}\right)\right) \partial_{i j} \tilde{u}_{J}\right|_{U_{4}^{m, 1}(0)}\right\|_{0, \alpha}+\sup _{U_{4}^{m, 1}(0)}\left|\tilde{u}_{J}-u^{\prime}\right|\right),
\end{aligned}
$$

whenever $J$ is large enough that $U_{6}^{m, 1}(0) \subset \tilde{\Omega}_{J} \times \tilde{I}_{J}$, and where the constant $C_{6}$ will depend on $m, n, \alpha, \kappa$. But both terms on the right-hand side converge to 0 as $J \rightarrow \infty$, since $\partial_{i j} \tilde{u}_{J}$ is bounded in $C^{0, \alpha}$ on compact subsets (by inequality (21)) and since $\left(\hat{g}^{i j}\left(D \tilde{u}_{J}\right)-\hat{g}^{i j}\left(D u^{\prime}\right)\right) \rightarrow 0$ in $C^{1}$ on compact sets. This means that, on $U_{2}^{m, 1}(0)$, the convergence $\tilde{u}_{J} \rightarrow u^{\prime}$ is $C^{2, \alpha}$. In particular, the terms of the $C^{2, \alpha}$ norm of $\tilde{u}_{J}$ involved in the definition of $G_{2, \alpha}\left(\tilde{M}_{J}, 0\right)$ will converge to 0 (since these terms are zero on $u^{\prime}$ ). This finally gives a contradiction because we dilated in such a way that $G_{2, \alpha}\left(\tilde{M}_{J}, 0\right)=1$, which implies that $\left[D \tilde{u}_{J}\right]_{\alpha}+\left\|D^{2} \tilde{u}_{J}\right\|_{0, \alpha}+\left\|\partial_{t} \tilde{u}_{J}\right\|_{0, \alpha}$ is bounded from below, independently of $J$, on $U_{2}^{m, 1}(0)$. Therefore $\bar{\epsilon}>0$.

Corollary 14. Let $\epsilon$ and $C_{5}$ be as in Theorem 13. Let $\mathcal{M}$ satisfy Assumption 2, with $X_{0} \in \mathcal{M}$ and $\tau\left(X_{0}\right)=\sup I .{ }^{11}$ Suppose that $\rho_{0}>\rho>0$ are such that $Q_{\rho_{0}}^{m, n, 1}(Y) \subset$ $\Omega \times \mathbb{R}^{n} \times I$ and

$$
\Theta\left(M, Y, s, \rho_{0}\right) \geq 1-\epsilon,
$$

${ }^{11}$ By these assumptions, the flow will be smooth at time $\tau\left(X_{0}\right)$, since we are taking $X_{0}$ to be a point on the flow. 
for all $Y \in Q_{\rho}^{m, n, 1}\left(X_{0}\right) \cap \mathcal{M}$ and all $s \in\left(\tau(Y)-\rho^{2}, \tau(Y)\right)$. Then

$$
\sup _{\mathcal{M} Q_{\rho}^{m, n, 1}\left(X_{0}\right)} G_{2, \alpha}(M, \cdot) d\left(\cdot, P_{\rho}^{m, n, 1}\left(X_{0}\right)\right) \leq C_{5} .
$$

Proof. This follows easily from Theorem 13 after taking the dilation $D_{1 / \rho}\left(\mu-X_{0}\right)$ and applying (17).

The next corollary should be compared to Theorem 3.5 of [White 2005].

Corollary 15. Let $\mathcal{M}$ satisfy Assumption 8. Let $X_{0}$ lie in the closure $\bar{M}$ such that $\tau\left(X_{0}\right)=\sup I .^{12}$ Suppose $\rho_{0}>\rho>0$ are such that $Q_{\rho_{0}}^{m, n, 1}(Y) \subset \Omega \times \mathbb{R}^{n} \times I$ and

$$
\Theta\left(M, Y, s, \rho_{0}\right) \geq 1-\epsilon,
$$

for all $Y \in Q_{\rho}^{m, n, 1}\left(X_{0}\right) \cap \mu$ and all $s \in\left(\tau(Y)-\rho^{2}, \tau(Y)\right)$. Then $\bar{M}$ will be smooth in some spacetime neighbourhood of $X_{0}$.

Proof. We take a sequence $X_{J} \rightarrow X_{0}$ (as $\left.J \rightarrow \infty\right)$ in $\mathcal{M}$ with $\tau\left(X_{J}\right)<\tau\left(X_{0}\right)$ and with $\hat{x}_{J}=\hat{x}_{0}$. For large $J,\left\|X_{J}-X_{0}\right\|<\rho / 2$ and we define

$$
M_{J}=\left\{Y \in M \mid \tau(Y) \leq \tau\left(X_{J}\right)\right\} .
$$

Now $\Theta\left(M_{J}, Y, s, \rho_{0}\right) \geq 1-\epsilon$ for $Y \in Q_{\rho / 2}^{m, n, 1}\left(X_{J}\right) \cap \mu_{J} \subset Q_{\rho}^{m, n, 1}\left(X_{0}\right) \cap M$ and $s \in\left(\tau(Y)-\rho^{2} / 4, \tau(Y)\right) \subset\left(\tau(Y)-\rho^{2}, \tau(Y)\right)$. Then, by Corollary 14,

$$
\sup _{\mathcal{M}_{J} \cap Q_{\rho / 2}^{m, n, 1}\left(X_{J}\right)} G_{2, \alpha}\left(\mathcal{M}_{J}, \cdot\right) d\left(\cdot, P_{\rho / 2}^{m, n, 1}\left(X_{J}\right)\right) \leq C_{5}
$$

for large $J$. This gives a $C^{2, \alpha}$ bound on each $\mu_{J}$ in some fixed spacetime neighbourhood of $X_{0}$. Then, since $\tau\left(X_{J}\right) \rightarrow \tau\left(X_{0}\right)$, we see that $\bar{M}$ is $C^{2, \alpha}$ in this neighbourhood and therefore smooth.

Theorem 16. Let $\mathcal{M}$ be a spacelike graphic mean curvature flow in $\mathbb{R}_{n}^{m+n} \times \mathbb{R}$, given by a smooth function $u: \Omega \times(0, T) \rightarrow \mathbb{R}^{n}$ with $\|D u\|^{2} \leq 1-\kappa$ for some positive constant $\kappa$. Then $M$ can be extended smoothly to the time $T$.

Proof. We can extend $u$ continuously to $T$, thanks to Proposition 17, and let $X_{0}=\left(\hat{x}_{0}, u\left(\hat{x}_{0}, T\right), T\right)$ for any $\hat{x}_{0} \in \Omega$. By Proposition $1, u$ satisfies system (5). We can take a convex, bounded neighbourhood $\Omega_{0} \subset \Omega$ of $\hat{x}_{0}$ and some $t_{0} \in(0, T)$. Then the flow $\mu_{0}$ given by the restriction of $u$ to $\Omega_{0} \times\left(t_{0}, T\right)$ will satisfy Assumption 8. Choosing $\rho_{0}>0$ to be sufficiently small, we first apply Theorem 18 and Proposition 12 to get $\lim _{t \rightarrow T} \Theta\left(M_{0}, X_{0}, t, \rho_{0}\right)>1-\epsilon$. Then we can apply Corollary 11 , which allows us to use Corollary 15 to get smoothness of $\bar{M}_{0}$ in a neighbourhood of $X_{0}$. We can do this at any $\hat{x}_{0} \in \Omega$, and therefore $\mu$ can be extended smoothly to $T$.

${ }^{12}$ By these assumptions, $\bar{M}$ is continuous at time $\tau\left(X_{0}\right)$ but not necessarily smooth, since we only assume $X_{0}$ to be on the closure and not necessarily on the flow itself. 


\section{Appendix}

Proposition 17. Suppose that $\mathcal{M}$ is as in Assumption 2, with $I=[a, b)$. Then $u$ can be extended to a continuous function on $\Omega \times[a, b]$.

Proof. Take the linear operator $P=\partial_{t}-\hat{g}^{i j}(D u) \partial_{i j}$. Using $P u=0$, applying Theorem 2.14 of [Lieberman 1996] (in particular, the comment that follows it) on cylinders in $\Omega \times(a, b)$ tells us that, for any $\hat{x} \in \Omega, u(\hat{x}, \cdot)$ is uniformly continuous on some interval with supremum $b$. It can therefore be extended continuously to $[a, b]$. On $\Omega \times[a, b)$ we have $|u(\hat{x}, t)-u(\hat{y}, t)| \leq(1-\kappa)^{1 / 2}|\hat{x}-\hat{y}|$, so taking the limit of this as $t \rightarrow b$ gives continuity of the extension with respect to $\hat{x}$.

Theorem 18. Suppose that $M$ satisfies Assumption 8 , with $I=(0, T)$. For any $X_{0}=\left(\hat{x}_{0}, u\left(\hat{x}_{0}, T\right), T\right)$ with $\hat{x}_{0} \in \Omega$, we will have $\lim _{t \rightarrow T} \Theta\left(M, X_{0}, t\right) \geq 1$.

It is important to remember that we are not assuming the flow to be smooth on $\Omega \times(0, T]$, only continuous. The proof of this theorem is roughly the same as the proofs of similar results in [Wang 2001].

Proof. We will first define a function on the flow, $\zeta=1+\log \left(1 / \kappa^{m / 2}\right)-\log (\cosh \theta)$, where $\theta$ is the hyperbolic angle defined on page 3 of [Li and Salavessa 2011]. ${ }^{13}$ An evolution equation discussed in Sections 4 and 5 of the same work tells us that

$$
\left(\frac{d}{d t}-\Delta_{M(t)}\right) \zeta \geq \kappa|B|^{2}
$$

where $|B|^{2}$ is the norm of the second fundamental form on the spatial slices. We note that there exist constants $C_{7}, C_{8}>0$ (depending on $\kappa$ ) such that $C_{7}|B|^{2} \leq$ $\left|D^{2} u\right|^{2} \leq C_{8}|B|^{2} .{ }^{14}$ Another useful fact is that, by the assumption $\|D u\| \|^{2} \leq 1-\kappa$, there exists a constant $C_{9}(\kappa)>0$ such that if $v \in \mathbb{R}_{n}^{m+n}$ is any tangent vector to $M(t)$ then $\langle v, v\rangle \leq|v|^{2} \leq C_{9}\langle v, v\rangle$. If we use $\phi_{\rho, X_{0}}$ from Definition 9 for small enough $\rho$, (11) gives

$$
\frac{d}{d t} \int_{\mathcal{M}(t)} \Phi_{X_{0}} \zeta \phi_{\rho, X_{0}} \geq \int_{\mathcal{M}(t)} \Phi_{X_{0}}\left(\frac{d}{d t}-\Delta_{\mathcal{M}(t)}\right)\left(\zeta \phi_{\rho, X_{0}}\right) .
$$

It is easy to check, as in [Ecker 2004, Lemma 3.14], that we have the product rule

$$
\begin{aligned}
& \left(\frac{d}{d t}-\Delta_{\mathcal{M}(t)}\right)\left(\phi_{\rho, X_{0}} \zeta\right) \\
& \quad=\zeta\left(\frac{d}{d t}-\Delta_{\mathcal{M}(t)}\right) \phi_{\rho, X_{0}}+\phi_{\rho, X_{0}}\left(\frac{d}{d t}-\Delta_{\mathcal{M}(t)}\right) \zeta-2\left\langle\operatorname{grad}_{\mathcal{M}(t)} \phi_{\rho, X_{0}}, \operatorname{grad}_{\mathcal{M}(t)} \zeta\right\rangle .
\end{aligned}
$$

${ }^{13}$ At a point on $M, \cosh \theta$ is just the value of $1 / \sqrt{\operatorname{det} \hat{g}}$ at the corresponding point in $\Omega \times I$.

${ }^{14}$ We can write $|B|^{2}=\left|\left\langle B_{i j}, B_{k l}\right\rangle \hat{g}^{i k} \hat{g}^{j l}\right|$; see [Li and Salavessa 2011] for details. $\left|D^{2} u\right|$ just denotes the Euclidean norm of $D^{2} u$, and to prove the inequality we need the fact that the eigenvalues of $D u^{T} D u$ are bounded above and below. Compare to [Ilmanen 1997, p. 31]. 
By Young's inequality,

$$
\begin{aligned}
\left\langle\operatorname{grad}_{\mathcal{M}(t)} \phi_{\rho, X_{0}}, \operatorname{grad}_{\mathcal{M}(t)} \zeta\right\rangle & =\left\langle\frac{\operatorname{grad}_{\mathcal{M}(t)} \phi_{\rho, X_{0}}}{\sqrt{\phi_{\rho, X_{0}}}}, \sqrt{\phi_{\rho, X_{0}}} \operatorname{grad}_{\mathcal{M}(t)} \zeta\right\rangle \\
& \leq \frac{1}{2 \epsilon} \frac{\left|\operatorname{grad}_{\mathcal{M}(t)} \phi_{\rho, X_{0}}\right|^{2}}{\phi_{\rho, X_{0}}}+\frac{\epsilon}{2} \phi_{\rho, X_{0}}\left|\operatorname{grad}_{\mathcal{M}(t)} \zeta\right|^{2} \\
& \leq \frac{1}{\epsilon} C_{10} \frac{\left|D \phi_{\rho, X_{0}}\right|^{2}}{\phi_{\rho, X_{0}}}+\frac{\epsilon}{2} C_{9} \phi_{\rho, X_{0}}\left\langle\operatorname{grad}_{\mathcal{M}(t)} \zeta, \operatorname{grad}_{\mathcal{M}(t)} \zeta\right\rangle
\end{aligned}
$$

where $C_{10}(\kappa)>0$ and $\epsilon$ is any positive number. Since $\phi_{\rho, X_{0}}$ is compactly supported on the flow, Example 3.16 of [Ecker 2004] ${ }^{15}$ implies that

$$
\left|D \phi_{\rho, X_{0}}\right|^{2} / \phi_{\rho, X_{0}} \leq 2 \max \left|D^{2} \phi_{\rho, X_{0}}\right|
$$

where we remember that $\left|D^{2} \phi_{\rho, X_{0}}\right|<C_{1} / \rho^{2}$. Using facts from [Li and Salavessa 2011] (see Equation 3.9 and the first inequality for $|B|^{2}$ in the proof of Proposition 5.2 there), we see that $\left\langle\operatorname{grad}_{\mathcal{M}(t)} \zeta, \operatorname{grad}_{\mathcal{M}(t)} \zeta\right\rangle \leq C_{11}|B|^{2}$ for some constant $C_{11}(\kappa)$. So there exist constants $C_{12}, C_{13}, C_{14}>0$ (depending on $\kappa, \rho$ ) such that

$$
\begin{array}{r}
2\left\langle\operatorname{grad}_{\mathcal{M}(t)} \phi_{\rho, X_{0}}, \operatorname{grad}_{\mathcal{M}(t)} \zeta\right\rangle \leq \frac{C_{12}}{\epsilon}+\epsilon C_{13} \phi_{\rho, X_{0}}|B|^{2}, \\
\left(\frac{d}{d t}-\Delta_{\mathcal{M}(t)}\right) \phi_{\rho, X_{0}} \leq C_{14},
\end{array}
$$

where we prove the second inequality as in Theorem 6. Combining all of the inequalities above,

$$
\begin{aligned}
\frac{d}{d t} \int_{\mathcal{M}(t)} \Phi_{X_{0}} \zeta \phi_{\rho, X_{0}} & \geq \int_{\mathcal{M}(t)} \Phi_{X_{0}}\left(\kappa \phi_{\rho, X_{0}}|B|^{2}-C_{15} C_{14}-\frac{C_{12}}{\epsilon}-\epsilon C_{13} \phi_{\rho, X_{0}}|B|^{2}\right) \\
& =\frac{\kappa}{2} \int_{\mathcal{M}(t)} \Phi_{X_{0}} \phi_{\rho, X_{0}}|B|^{2}-C_{16} \Theta\left(\mathcal{M}, X_{0}, t\right),
\end{aligned}
$$

where we use the fact that $\zeta$ is clearly less than or equal to some constant $C_{15}(\kappa)$ and choose $\epsilon=\kappa / 2 C_{13}$ and $C_{16}=C_{15} C_{14}+C_{12} / \epsilon$. We can use this to prove the theorem. We assume that $\lim _{t \rightarrow T} \Theta\left(\mathcal{M}, X_{0}, t\right)<1$ and hope to get a contradiction. So for $t$ close enough to $\tau\left(X_{0}\right)=T$ (say $t \in(T-\delta, T)$ for some $\delta>0$ ) we can assume that

$$
\frac{d}{d t} \int_{\mathcal{M}(t)} \Phi_{X_{0}} \zeta \phi_{\rho, X_{0}} \geq \frac{\kappa}{2} \int_{\mathcal{M}(t)} \Phi_{X_{0}} \phi_{\rho, X_{0}}|B|^{2}-C_{16}
$$

We can see how this inequality is affected by parabolic dilations, $D_{\lambda}$ for $\lambda>1$, by using the transformation formula for integrals, and by noting that $\zeta$ involves first

${ }^{15}|D \phi|^{2} / \phi \leq 2 \max \left|D^{2} \phi\right|$ for compactly supported $C^{2}$ functions. 
derivatives and $|B|$ involves second derivatives. We get

$$
\frac{d}{d s} \int_{D_{\lambda}\left(\mathcal{M}-X_{0}\right)(s)} \Phi_{0} \zeta \phi_{\lambda \rho, 0} \geq-\frac{C_{16}}{\lambda^{2}}+\frac{\kappa}{2} \int_{D_{\lambda}\left(\mathcal{M}-X_{0}\right)(s)} \Phi_{0}|B|^{2} \phi_{\lambda \rho, 0}
$$

for $s \in\left(-\lambda^{2} \delta, 0\right)$, remembering that $\lambda>1$. We now take $\tau<\delta / 2$ and integrate with respect to $s$ over the interval $(-\delta / 2-\tau,-\delta / 2)$ to get

$$
\left[\int_{D_{\lambda}\left(\mathcal{M}-X_{0}\right)(s)} \Phi_{0} \zeta \phi_{\lambda \rho, 0}\right]_{-\delta / 2-\tau}^{-\delta / 2} \geq-\frac{C_{16} \tau}{\lambda^{2}}+\frac{\kappa}{2} \int_{-\delta / 2-\tau}^{-\delta / 2} \int_{D_{\lambda}\left(\mathcal{M}-X_{0}\right)(s)} \Phi_{0}|B|^{2} \phi_{\lambda \rho, 0} .
$$

The left-hand side and the first term on the right-hand side clearly have limit zero as $\lambda \rightarrow \infty$. Therefore we must have $\int_{-\delta / 2-\tau}^{-\delta / 2} \int_{D_{\lambda}\left(\mathcal{M}-X_{0}\right)(s)} \Phi_{0}|B|^{2} \phi_{\lambda \rho, 0} \rightarrow 0$. As in [Wang 2001, p. 26], we can use the integral mean value theorem to choose sequences $\lambda_{J} \rightarrow \infty, \tau_{J} \rightarrow 0$ and $s_{J} \in\left[-\delta / 2-\tau_{J},-\delta / 2\right]$ such that

$$
\int_{D_{\lambda_{J}}\left(\mathcal{M}-X_{0}\right)\left(s_{J}\right)} \Phi_{0}|B|^{2} \phi_{\lambda_{J} \rho, 0} \rightarrow 0 \text { as } J \rightarrow \infty .
$$

We have $\delta / 2 \leq\left|s_{J}\right| \leq \delta$, so

$$
\Phi_{0}\left(\hat{x}, \tilde{x}, s_{J}\right)=\frac{\exp \left(\left(-|\hat{x}|^{2}+|\tilde{x}|^{2}\right) / 4\left|s_{J}\right|\right)}{\left(4 \pi\left|s_{J}\right|\right)^{m / 2}} \geq \frac{\exp \left(-|\hat{x}|^{2} / 2 \delta\right)}{(4 \pi \delta)^{m / 2}} .
$$

The function $\phi_{\lambda_{J} \rho, 0}$ is zero outside $B_{\lambda_{J} \rho}^{m}(0) \times \mathbb{R}^{n}$ and equals 1 inside $B_{\lambda_{J} \rho / 2}^{m}(0) \times \mathbb{R}^{n}$. For any $R>0$ we can take $J$ large enough that $B_{R}^{m}(0) \times \mathbb{R}^{n} \subset B_{\lambda_{J} \rho / 2}^{m}(0) \times \mathbb{R}^{n}$, implying that

$$
\frac{\exp \left(-R^{2} / 2 \delta\right)}{(4 \pi \delta)^{m / 2}} \int_{D_{\lambda_{J}}\left(\mathcal{M}-X_{0}\right)\left(s_{J}\right) \cap B_{R}^{m}(0) \times \mathbb{R}^{n}}|B|^{2} \leq \int_{D_{\lambda_{J}}\left(\mathcal{M}-X_{0}\right)\left(s_{J}\right)} \Phi_{0} \phi_{\rho \lambda_{J}, 0}|B|^{2} .
$$

We therefore have

$$
\frac{\exp \left(-R^{2} / 2 \delta\right)}{(4 \pi \delta)^{m / 2}} \int_{D_{\lambda_{J}}\left(\mathcal{M}-X_{0}\right)\left(s_{J}\right) \cap B_{R}^{m}(0) \times \mathbb{R}^{n}}|B|^{2} \rightarrow 0 \quad \text { as } J \rightarrow \infty .
$$

Now let us consider the functions $\tilde{u}_{J}(\hat{x})$ whose graphs give the spatial slices $D_{\lambda_{J}}\left(M-X_{0}\right)\left(s_{J}\right)$. The fact that $\lambda_{J} \rightarrow \infty$ tells us that, for any $R>0$, we can take $J$ large enough that $B_{R}^{m}(0)$ is contained in the domain of $\tilde{u}_{J}$. Since we also have a uniform bound on the gradients $D \tilde{u}_{J}$, the usual Arzelà-Ascoli theorem argument gives a subsequence (which we continue to denote by $\tilde{u}_{J}$ ) converging pointwise on $\mathbb{R}^{m}$, and uniformly on each $B_{R}^{m}(0)$, to some limit $\tilde{u}$. Define

$$
v_{J}^{k \gamma}=\partial_{k} \tilde{u}_{J}^{\gamma} \quad \text { and } \quad c_{J}^{k \gamma}=\frac{1}{\operatorname{vol}\left(B_{R}^{m}(0)\right)} \int_{B_{R}^{m}(0)} v_{J}^{k \gamma},
$$


and take a convergent subsequence $c_{J}^{k \gamma} \rightarrow c^{k \gamma}$ (since the sequence is bounded, by the gradient bound on $\tilde{u}_{J}$ ). Apply the Poincaré inequality to get

$$
\int_{B_{R}^{m}(0)}\left|v_{J}^{k \gamma}-c_{J}^{k \gamma}\right|^{2} \leq C_{17} \int_{B_{R}^{m}(0)}\left|D v_{J}^{k \gamma}\right|^{2} \leq C_{17} \int_{B_{R}^{m}(0)}\left|D^{2} \tilde{u}_{J}\right|^{2} \rightarrow 0,
$$

where the last step uses (24) and $\left|D^{2} \tilde{u}_{J}\right|^{2} \leq C_{8}|B|^{2}$. So $v_{J}^{k \gamma}-c_{J}^{k \gamma} \rightarrow 0$ with respect to the $L^{2}$ norm on $B_{R}^{m}(0)$. Now we can assume that the derivatives of our sequence converge pointwise almost everywhere to constants. These constants will be the weak derivatives of $\tilde{u}$, which therefore must be linear. Since this holds for any $R$, and since $\Theta$ is equal to 1 on nonmoving planes, we can use this to apply the dominated convergence theorem to see that

$$
\begin{aligned}
1 & \leq \lim _{J \rightarrow \infty} \Theta\left(D_{\lambda_{J}}\left(\mathcal{M}-X_{0}\right), 0, s_{J}\right) \\
& =\lim _{J \rightarrow \infty} \Theta\left(M, X_{0}, T+s_{J} / \lambda_{J}^{2}\right)=\lim _{t \rightarrow T} \Theta\left(M, X_{0}, t\right),
\end{aligned}
$$

contradicting the assumption that $\lim _{t \rightarrow T} \Theta\left(\mathcal{M}, X_{0}, t\right)<1$.

We only need the next proposition in the proof of Theorem 13. First we note that a bound on $G_{2, \alpha}$ on some subset of spacetime implies a bound on $[D u]_{\alpha}+$ $\left[D^{2} u\right]_{\alpha}+\sup \left|D^{2} u\right|+\left[\partial_{t} u\right]_{\alpha}+\sup \left|\partial_{t} u\right|$ on a subset of $\mathbb{R}^{m, 1}$. If the flow contains the origin, then inequality (4) and the spacelike assumption give a bound on $|D u|$ and on $|u|$ on this subset, and therefore a bound on $\|u\|_{2, \alpha}$.

Proposition 19. The sequence $\tilde{M}_{J} \cap\{X \mid \tau(X) \leq 0\}$, from the proof of Theorem 13, has a convergent subsequence (this is parabolic $C^{2}$ convergence on compact subsets). The limit is a smooth entire flow $\mathcal{M}^{\prime}$, defined on $\mathbb{R}^{m} \times(-\infty, 0]$, satisfying Assumption 2, with uniformly bounded mean curvature vector.

Proof. Let $\tilde{u}_{J}, \tilde{M}_{J}$, etc. be exactly as in the proof of Theorem 13. Then, since $\lambda_{J} \rightarrow \infty$ and $\sup \tilde{I}_{J} \geq 0$, any compact subset of $\mathbb{R}^{m} \times(-\infty, 0]$ will be contained in the domain of $\tilde{u}_{J}$ for large enough $J$. By inequality $(21), G_{2, \alpha}\left(\tilde{\mathcal{M}}_{J}, \cdot\right)$ will be uniformly bounded on compact subsets of spacetime with $\tau(X) \leq 0$, as $J \rightarrow \infty$. Therefore we get uniform bounds on $\left\|\tilde{u}_{J}\right\|_{2, \alpha}$ on compact subsets of $\mathbb{R}^{m} \times(-\infty, 0]$. We can use this to prove convergence of a subsequence by following the same steps as in the proof of the Arzelà-Ascoli theorem. We use the Cantor diagonalization process to choose a pointwise convergent subsequence on $\mathbb{R}^{m} \times(-\infty, 0]$, and then the $C^{2, \alpha}$ estimates imply $C^{2}$ convergence on compact subsets. The limit $\mathcal{M}^{\prime}$ is clearly $C^{2, \alpha}$ and therefore smooth by the usual induction argument (since the system (5) holds on $\mathcal{M}^{\prime}$ ). Also, the $C^{2, \alpha}$ bound implies a uniform bound on the mean curvature of $\mathcal{M}^{\prime}$. 


\section{References}

[Ecker 2004] K. Ecker, Regularity theory for mean curvature flow, Progress in Nonlinear Differential Equations and their Applications 57, Birkhäuser, Boston, 2004. MR 2005b:53108 Zbl 1058.53054

[Friedman 1964] A. Friedman, Partial differential equations of parabolic type, Prentice-Hall, Englewood Cliffs, NJ, 1964. MR 31 \#6062 Zbl 0144.34903

[Huisken 1990] G. Huisken, "Asymptotic behavior for singularities of the mean curvature flow", $J$. Differential Geom. 31:1 (1990), 285-299. MR 90m:53016 Zbl 0694.53005

[Ilmanen 1997] T. Ilmanen, "Singularities of mean curvature flow of surfaces", preprint, 1997, Available at www.math.ethz.ch/ ilmanen/papers/sing.ps.

[Krylov 1996] N. V. Krylov, Lectures on elliptic and parabolic equations in Hölder spaces, Graduate Studies in Math. 12, American Mathematical Society, Providence, RI, 1996. MR 97i:35001 Zbl 0865.35001

[Lee 2003] J. M. Lee, Introduction to smooth manifolds, Graduate Texts in Math. 218, Springer, New York, 2003. MR 2003k:58001 Zbl 1030.53001

[Li and Salavessa 2011] G. Li and I. Salavessa, "Mean curvature flow of spacelike graphs", Math. Z. 269:3-4 (2011). Zbl 1231.53057

[Lieberman 1996] G. M. Lieberman, Second order parabolic differential equations, World Scientific, River Edge, NJ, 1996. MR 98k:35003 Zbl 0884.35001

[O’Neill 1983] B. O’Neill, Semi-Riemannian geometry, with applications to relativity, Pure and Applied Mathematics 103, Academic Press, New York, 1983. MR 85f:53002 Zbl 0531.53051

[Thorpe 2011] B. S. Thorpe, "The maximal graph Dirichlet problem in semi-Euclidean spaces", preprint, 2011. To appear in Comm. Anal. Geom. arXiv 1112.4219

[Wang 2001] M.-T. Wang, "Mean curvature flow of surfaces in Einstein four-manifolds", J. Differential Geom. 57:2 (2001), 301-338. MR 2003j:53108 Zbl 1035.53094

[White 2005] B. White, "A local regularity theorem for mean curvature flow", Ann. of Math. (2) 161:3 (2005), 1487-1519. MR 2006i:53100 Zbl 1091.53045

Received March 19, 2011. Revised July 11, 2011.

BENJAMIN STUART THORPE

DEPARTMENT OF MATHEMATICAL SCIENCES

DURHAM UNIVERSITY

SCIENCE LABORATORIES

SOUTH ROAD

DURHAM, DH1 3LE

UNITED KINGDOM

b.s.thorpe@durham.ac.uk 


\title{
PACIFIC JOURNAL OF MATHEMATICS
}

\author{
http://pacificmath.org \\ Founded in 1951 by \\ E. F. Beckenbach (1906-1982) and F. Wolf (1904-1989)
}

\section{EDITORS}

V. S. Varadarajan (Managing Editor)

Department of Mathematics

University of California

Los Angeles, CA 90095-1555

pacific@math.ucla.edu

Vyjayanthi Chari

Department of Mathematics

University of California

Riverside, CA 92521-0135

chari@math.ucr.edu

\section{Robert Finn}

Department of Mathematics Stanford University

Stanford, CA 94305-2125

finn@math.stanford.edu

Kefeng Liu

Department of Mathematics

University of California

Los Angeles, CA 90095-1555

liu@math.ucla.edu
Darren Long

Department of Mathematics

University of California

Santa Barbara, CA 93106-3080

long@math.ucsb.edu

Jiang-Hua Lu

Department of Mathematics

The University of Hong Kong

Pokfulam Rd., Hong Kong jhlu@maths.hku.hk

Alexander Merkurjev

Department of Mathematics

University of California

Los Angeles, CA 90095-1555

merkurev@math.ucla.edu
Sorin Popa

Department of Mathematics University of California

Los Angeles, CA 90095-1555 popa@math.ucla.edu

Jie Qing

Department of Mathematics

University of California

Santa Cruz, CA 95064

qing@cats.ucsc.edu

Jonathan Rogawski

Department of Mathematics

University of California

Los Angeles, CA 90095-1555

jonr@math.ucla.edu

\section{PRODUCTION}

pacific@math.berkeley.edu

\section{SUPPORTING INSTITUTIONS}

ACADEMIA SINICA, TAIPEI

CALIFORNIA INST. OF TECHNOLOGY INST. DE MATEMÁTICA PURA E APLICADA KEIO UNIVERSITY

MATH. SCIENCES RESEARCH INSTITUTE NEW MEXICO STATE UNIV.

OREGON STATE UNIV.

\author{
STANFORD UNIVERSITY \\ UNIV. OF BRITISH COLUMBIA \\ UNIV. OF CALIFORNIA, BERKELEY \\ UNIV. OF CALIFORNIA, DAVIS \\ UNIV. OF CALIFORNIA, LOS ANGELES \\ UNIV. OF CALIFORNIA, RIVERSIDE \\ UNIV. OF CALIFORNIA, SAN DIEGO \\ UNIV. OF CALIF., SANTA BARBARA
}

\author{
UNIV. OF CALIF., SANTA CRUZ \\ UNIV. OF MONTANA \\ UNIV. OF OREGON \\ UNIV. OF SOUTHERN CALIFORNIA \\ UNIV. OF UTAH \\ UNIV. OF WASHINGTON \\ WASHINGTON STATE UNIVERSITY
}

These supporting institutions contribute to the cost of publication of this Journal, but they are not owners or publishers and have no responsibility for its contents or policies.

See inside back cover or pacificmath.org for submission instructions.

The subscription price for 2012 is US \$420/year for the electronic version, and \$485/year for print and electronic.

Subscriptions, requests for back issues from the last three years and changes of subscribers address should be sent to Pacific Journal of Mathematics, P.O. Box 4163, Berkeley, CA 94704-0163, U.S.A. Prior back issues are obtainable from Periodicals Service Company, 11 Main Street, Germantown, NY 12526-5635. The Pacific Journal of Mathematics is indexed by Mathematical Reviews, Zentralblatt MATH, PASCAL CNRS Index, Referativnyi Zhurnal, Current Mathematical Publications and the Science Citation Index.

The Pacific Journal of Mathematics (ISSN 0030-8730) at the University of California, c/o Department of Mathematics, 969 Evans Hall, Berkeley, CA 94720-3840, is published monthly except July and August. Periodical rate postage paid at Berkeley, CA 94704, and additional mailing offices. POSTMASTER: send address changes to Pacific Journal of Mathematics, P.O. Box 4163, Berkeley, CA 94704-0163.

PJM peer review and production are managed by EditFLOW ${ }^{\mathrm{TM}}$ from Mathematical Sciences Publishers.

PUBLISHED BY PACIFIC JOURNAL OF MATHEMATICS

at the University of California, Berkeley 94720-3840

A NON-PROFIT CORPORATION

Typeset in LATEX

Copyright $(02012$ by Pacific Journal of Mathematics 


\section{PACIFIC JOURNAL OF MATHEMATICS}

Volume $255 \quad$ No. $2 \quad$ February 2012

On the local Langlands correspondences of DeBacker-Reeder and

Reeder for $\operatorname{GL}(\ell, F)$, where $\ell$ is prime

MOSHE ADRIAN

$R$-groups and parameters

281

DUBRAVKA BAN and DAVID GOLDBERG

Finite-volume complex-hyperbolic surfaces, their toroidal

305

compactifications, and geometric applications

LUCA FABRIZIO Di CERBO

Character analogues of Ramanujan-type integrals involving the Riemann 317 $\Xi$-function

ATUl DiXIT

Spectral theory for linear relations via linear operators

DANA GHEORGHE and FLORIAN-HORIA VASILESCU

Homogeneous links and the Seifert matrix

PEDRO M. GonZÁlez MANCHÓN

Quantum affine algebras, canonical bases, and $q$-deformation of arithmetical functions

HENRY H. KIM and KYU-HWAN LEE

Dirichlet-Ford domains and arithmetic reflection groups

GRANT S. LAKELAND

Formal equivalence of Poisson structures around Poisson submanifolds

IOAN MĂRCUT,

A regularity theorem for graphic spacelike mean curvature flows

BENJAMIN STUART THORPE

Analogues of level- $N$ Eisenstein series 